\title{
Quantum Computing and the Jones Polynomial
}

\author{
Louis H. Kauffman
}

\begin{abstract}
This paper is an exploration of relationships between the Jones polynomial and quantum computing. We discuss the structure of the Jones polynomial in relation to representations of the Temperley Lieb algebra, and give an example of a unitary representation of the braid group. We discuss the evaluation of the polynomial as a generalized quantum amplitude and show how the braiding part of the evaluation can be construed as a quantum computation when the braiding representation is unitary. The question of an efficient quantum algorithm for computing the whole polynomial remains open.
\end{abstract}

\section{Contents}

1. Introduction

2. Dirac Brackets

3. Braiding, Projectors and the Temperley Lieb Algebra

4. The Bracket Polynomial

5. Knot Amplitudes

6. Quantum Computing

7. Summary

References

\section{Introduction}

This paper is an exploration of issues interrelating the Jones polynomial [10] and quantum computing. In section 2 of the paper we review the formalism of Dirac brackets and some of the quantum physics associated with this formalism. The section ends with a brief description of the concept of quantum computer that we shall use in this paper. In section 3 we discuss the Jones and Temperley Lieb algebras and how they can be used to produce representations of the Artin

2000 Mathematics Subject Classification. Primary 81P68; Secondary 81-01.

Key words and phrases. Quantum computing, quantum topology, Jones polynomial.

Research on this paper was partially supported by National Science Foundation Grant DMS 9802859, and by the Defense Advanced Research Projects Agency (DARPA) and Air Force Materiel Command USAF under agreement number F30602-01-0522.

(C)2002 American Mathematical Society 
Braid group. While most of these representations are not unitary, we show how to construct non-trivial unitary representations of the three-strand braid group by considering the structure of two projectors. It turns out that two elementary projectors naturally generate a Temperley Lieb algebra. This provides a way to make certain unitary representations and to motivate the construction of both the Alexander and the Jones polynomial. In regard to the Alexander polynomial, we end this section with a representation of the Artin Braid Group, constructed using projectors, that is equivalent to the classical Burau representation. In section 4 we construct the bracket polynomial model for the Jones polynomial and relate its structure to the representations discussed in the previous section. Section 5 shows how to reformulate the bracket state sum in terms of discrete quantum amplitudes. This sets the stage for our proposal, explained in section 6 , for regarding knot invariants as quantum computers. This proposal needs unitary braiding (a special condition) and the results of the computer are probabilistic. Nevertheless, I believe that this model deserves consideration. The dialogue between topology and quantum computing is just beginning.

\section{Dirac Brackets}

We begin with a discussion of Dirac's notation, $\langle b \mid a\rangle$, [4]. In this notation $<a \mid$ and $|b\rangle$ are covectors and vectors respectively. $\langle b \mid a\rangle$ is the evaluation of $\mid a>$ by $<b \mid$, hence it is a scalar, and in ordinary quantum mechanics it is a complex number. One can think of this as the amplitude for the state to begin in " $a$ " and end in " $b$ ". That is, there is a process that can mediate a transition from state $a$ to state $b$. Except for the fact that amplitudes are complex valued, they obey the usual laws of probability. This means that if the process can be factored into a set of all possible intermediate states $c_{1}, c_{2}, \ldots, c_{n}$, then the amplitude for $a \longrightarrow b$ is the sum of the amplitudes for $a \longrightarrow c_{i} \longrightarrow b$. Meanwhile, the amplitude for $a \longrightarrow c_{i} \longrightarrow b$ is the product of the amplitudes of the two subconfigurations $a \longrightarrow c_{i}$ and $c_{i} \longrightarrow b$. Formally we have

$$
<b\left|a>=\Sigma_{i}<b\right| c_{i}><c_{i} \mid a>
$$

where the summation is over all the intermediate states $i=1, \ldots, n$.

In general, the amplitude for mutually disjoint processes is the sum of the amplitudes of the individual processes. The amplitude for a configuration of disjoint processes is the product of their individual amplitudes.

Dirac's division of the amplitudes into bras $\langle b|$ and kets $\mid a>$ is done mathematically by taking a vector space $V$ (a Hilbert space, but it can be finite dimensional) for the kets: $\mid a>$ belongs to $V$. The dual space $V^{*}$ is the home of the bras. Thus $<b \mid$ belongs to $V^{*}$ so that $<b \mid$ is a linear mapping $<b \mid: V \longrightarrow C$ where $C$ denotes the complex numbers. We restore symmetry to the definition by realizing that an element of a vector space $V$ can be regarded as a mapping from the complex numbers to $V$. Given $\mid a>: C \longrightarrow V$, the corresponding element of $V$ is the image of 1 (in $C$ ) under this mapping. In other words, $\mid a>(1)$ is a member of $V$. Now we have $\mid a>: C \longrightarrow V$ and $\langle b|: V \longrightarrow C$. The composition $\langle b|\circ| a\rangle=\langle b| a>: C \longrightarrow C$ is regarded as an element of $C$ by taking the specific value $\langle b \mid a\rangle$ (1). The complex numbers are regarded as the "vacuum", and the entire amplitude $\langle b \mid a\rangle$ is a "vacuum to vacuum" amplitude for a process that 
includes the creation of the state $a$, its transition to $b$, and the annihilation of $b$ to the vacuum once more.

Dirac notation has a life of its own. Let

$$
P=|y><x| .
$$

Let

$$
<x|| y>=<x \mid y>
$$

Then

$$
P P=|y><x||y><x|=|y><x| y><x|=<x| y>P .
$$

Up to a scalar multiple, $P$ is a projection operator. That is, if we let

$$
Q=P /<x \mid y>,
$$

then

$$
Q Q=P P /<x|y><x| y>=<x|y>P /<x| y><x|y>=P /<x| y>=Q .
$$

Thus $Q Q=Q$. In this language, the completeness of intermediate states becomes the statement that a certain sum of projections is equal to the identity: Suppose that $\Sigma_{i}\left|c_{i}><c_{i}\right|=1$ (summing over $i$ ) with $<c_{i} \mid c_{i}>=1$ for each $i$. Then

$$
\begin{gathered}
<b|a>=<b||a>=<b| \Sigma_{i}\left|c_{i}><c_{i}\right|\left|a>=\Sigma_{i}<b\right| \mid c_{i}><c_{i} \| a> \\
<b\left|a>=\Sigma_{i}<b\right| c_{i}><c_{i} \mid a>
\end{gathered}
$$

Iterating this principle of expansion over a complete set of states leads to the most primitive form of the Feynman integral [5]. Imagine that the initial and final states $a$ and $b$ are points on the vertical lines $x=0$ and $x=n+1$ respectively in the $x-y$ plane, and that $\left(c(k)_{i(k)}, k\right)$ is a given point on the line $x=k$ for $0<i(k)<m$. Suppose that the sum of projectors for each intermediate state is complete. That is, we assume that following sum is equal to one, for each $k$ from 1 to $n-1$ :

$$
\left|c(k)_{1}><c(k)_{1}\right|+\ldots+\left|c(k)_{m}><c(k)_{m}\right|=1 .
$$

Applying the completeness iteratively, we obtain the following expression for the amplitude $\langle b \mid a\rangle$ :

$$
<b|a>=\Sigma \Sigma \Sigma \ldots \Sigma<b| c(1)_{i(1)}><c(1)_{i(1)}\left|c(2)_{i(2)}>\ldots<c(n)_{i(n)}\right| a>
$$

where the sum is taken over all $i(k)$ ranging between 1 and $m$, and $k$ ranging between 1 and $n$. Each term in this sum can be construed as a combinatorial path from $a$ to $b$ in the two dimensional space of the $x-y$ plane. Thus the amplitude for going from $a$ to $b$ is seen as a summation of contributions from all the "paths" connecting $a$ to $b$. See Figure 1 . 

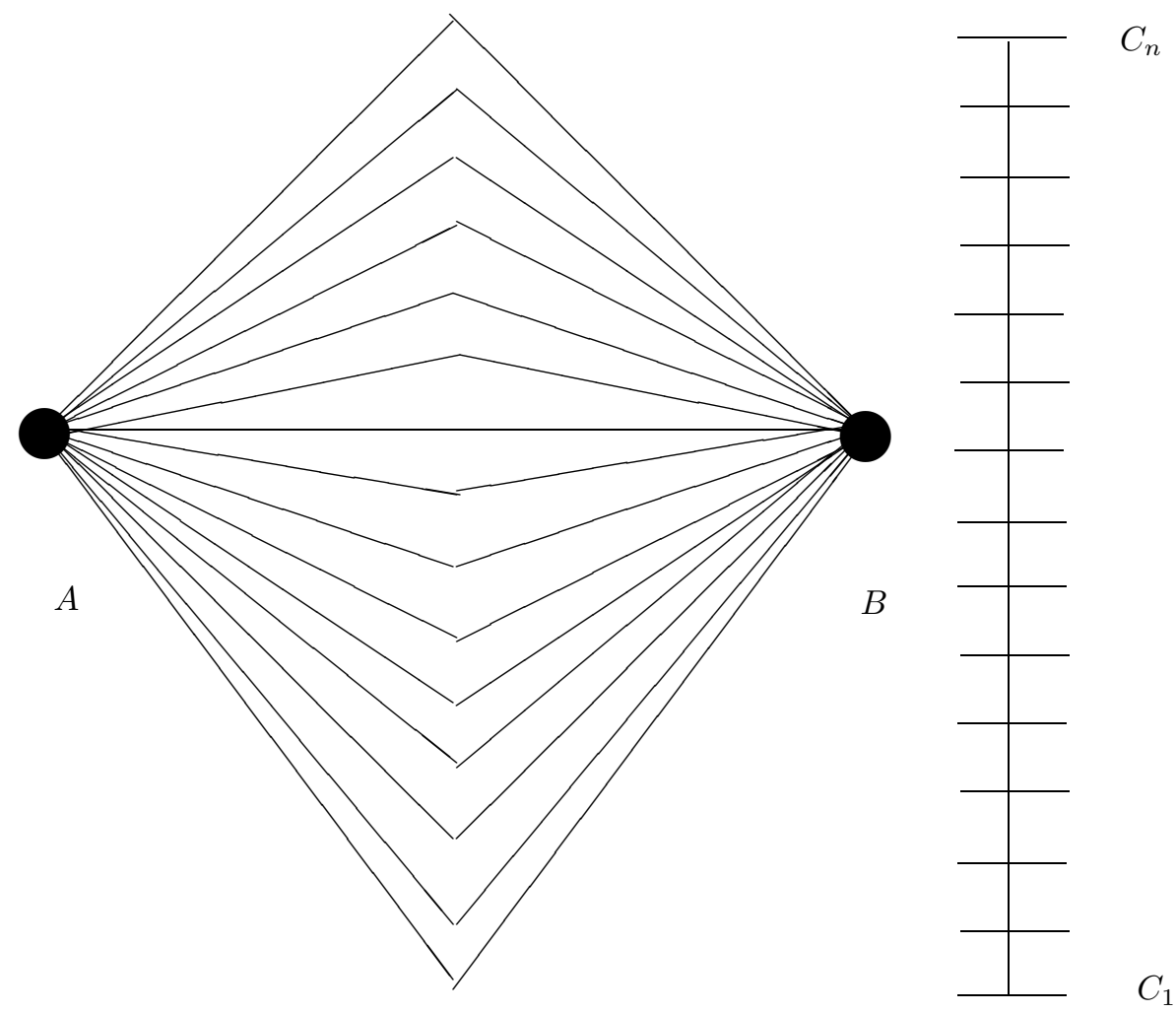

Figure 1 - Intermediates

Feynman used this description to produce his famous path integral expression for amplitudes in quantum mechanics. His path integral takes the form

$$
\int d \operatorname{Pexp}(i S)
$$

where $i$ is the square root of minus one, the integral is taken over all paths from point $a$ to point $b$, and $S$ is the action for a particle to travel from $a$ to $b$ along a given path. For the quantum mechanics associated with a classical (Newtonian) particle the action $S$ is given by the integral along the given path from $a$ to $b$ of the difference $T-V$ where $T$ is the classical kinetic energy and $V$ is the classical potential energy of the particle.

2.1. What is a Quantum Computer? We are now in a position to explain the definition of quantum computer that will be used in this paper. Let $H$ be a given finite dimensional vector space over the complex numbers $C$. Let $\left\{W_{0}, W_{1}, \ldots, W_{n}\right\}$ be an orthonormal basis for $H$ so that with $|i>:=| W_{i}>$ denoting $W_{i}$ and $<i \mid$ denoting the conjugate transpose of $|i\rangle$, we have

$$
<i \mid j>=\delta_{i j}
$$


where $\delta_{i j}$ denotes the Kronecker delta (equal to one when its indices are equal to one another, and equal to zero otherwise). Given a vector $v$ in $H$ let $|v|^{2}:=\langle v \mid v\rangle$. Note that $<i \mid v$ is the $i$-th coordinate of $v$.

An observation of $v$ returns one of the coordinates $\mid i>$ of $v$ with probability $\mid<$ $i|v|^{2}$. This model of observation is a simple instance of the situation with a quantum mechanical system that is in a mixed state until it is observed. The result of observation is to put the system into one of the basis states.

When the dimension of the space $H$ is two $(n=1)$, a vector in the space is called a qubit. A qubit represents one quantum of binary information. On observation, one obtains either the ket $\mid 0>$ or the ket $\mid 1>$. This constitutes the binary distinction that is inherent in a qubit. Note however that the information obtained is probabilistic. If the qubit is

$$
\psi=\alpha|0>+\beta| 1>,
$$

then the ket $\mid 0>$ is observed with probability $|\alpha|^{2}$, and the ket $\mid 1>$ is observed with probability $|\beta|^{2}$. In speaking of an idealized quantum computer, we do not specify the nature of measurement process beyond these probability postulates.

In the case of general dimension $n$ of the space $H$, we will call the vectors in $H$ qunits. It is quite common to use spaces $H$ that are tensor products of twodimensional spaces (so that all computations are expressed in terms of qubits) but this is not necessary in principle. One can start with a given space, and later work out factorizations into qubit transformations.

A quantum computation consists in the application of a unitary transformation $U$ to an initial qunit $\psi=a_{0}\left|0>+\ldots+a_{n}\right| n>$ with $|\psi|^{2}=1$, plus an observation of $U \psi$. An observation of $U \psi$ returns the ket $\mid i>$ with probability $|U \psi|^{2}$. In particular, if we start the computer in the state $|i\rangle$, then the probability that it will return the state $|j\rangle$ is $|\langle j|U| i\rangle|^{2}$.

It is the necessity for writing a given computation in terms of unitary transformations, and the probabilistic nature of the result that characterizes quantum computation. Such computation could be carried out by an idealized quantum mechanical system. It is hoped that such systems can be physically realized.

\section{Braiding, Projectors and the Temperley Lieb Algebra}

The Jones polynomial is one of the great mathematical breakthroughs of the twentieth century, and like many such breakthroughs it appears basically simple in retrospect. I will tell two stories in this section. The first story is a capsule summary of how Jones discovered the polynomial by way of an apparently strange algebraic structure that first appeared in his research on von Neumann algebras, and then was pointed out to be an algebra known to experts in the Potts model in statistical mechanics. The second story shows that the essential algebra for the needed representation of the braid group is present in the algebra generated by any two simple projectors (see below for the definitions of these terms) and that it is graphically illustrated by the Dirac bra-ket notation for these operators. 
Jones was studying the inclusion of one von Neumann algebra $N$ in another one $M$. In this context there is a projection $e_{1}: M \longrightarrow N$ so that the restriction of $e$ to $N$ is the identity mapping, and so that $e_{1}^{2}=e_{1}$. In his context the algebra $M$ could be extended to include this projector to an algebra $M_{1}=M \bigcup\left\{e_{1}\right\}$. Then we have

$$
N \subset M \subset M_{1}
$$

and the construction can be continued inductively to produce

$$
N \subset M \subset M_{1} \subset M_{2} \subset M_{3} \subset \ldots
$$

and an algebra of projectors

$$
e_{1}, e_{2}, e_{3}, \ldots
$$

such that

$$
\begin{gathered}
e_{i}^{2}=e_{i}, i=1,2,3, \ldots \\
e_{i} e_{i \pm 1} e_{i}=\kappa e_{i}, i=2,3, \ldots \\
e_{i} e_{j}=e_{j} e_{i},|i-j|>1 .
\end{gathered}
$$

We will call an algebra that can be expressed with generators and relations as above a Jones algebra. $J_{\infty}$ will denote a Jones algebra on infinitely many generators as above. $J_{n}$ will denote the Jones algebra generated by an identity element 1 and generators $e_{1}, \ldots, e_{n-1}$.

It was pointed out that the relations

$$
\begin{gathered}
e_{i} e_{i \pm 1} e_{i}=\kappa e_{i}, i=2,3, \ldots \\
e_{i} e_{j}=e_{j} e_{i},|i-j|>1
\end{gathered}
$$

look suspiciously like the basic braiding relations in the Artin Braid group which read

$$
\begin{gathered}
\sigma_{i} \sigma_{i \pm 1} \sigma_{i}=\sigma_{i \pm 1} \sigma_{i} \sigma_{i \pm 1}, i=2,3, \ldots \\
\sigma_{i} \sigma_{j}=\sigma_{j} \sigma_{i},|i-j|>1
\end{gathered}
$$




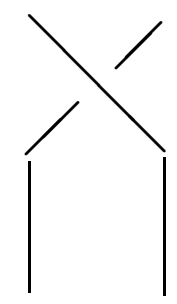

$\sigma_{1}$

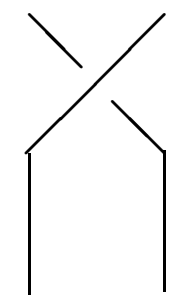

$\sigma_{1}^{-1}$

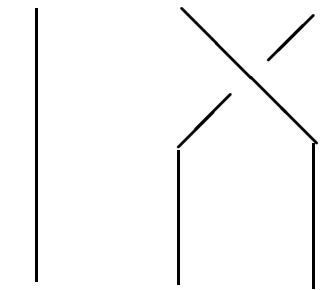

$\sigma_{2}$

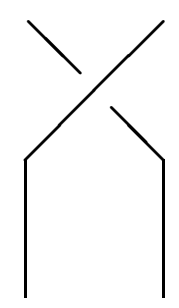

$\sigma_{2}^{-1}$

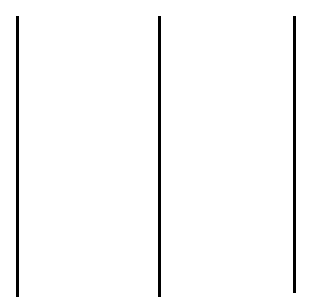

1

Figure 2 - Braid Group Generators

This pattern led Jones to first construct a representation of the Artin Braid Group to his algebra, and then to discover an invariant of knots and links that is related to this representation. Figure 2 illustrates the generators of the braid group. The second and third Reidemeister moves shown in Figure 6 illustrate the braiding relations except for commutativity of distant generators.

The representation that Jones discovered is a linear one in the form of

$$
\rho: B_{\infty} \longrightarrow J_{\infty}
$$

where

$$
\rho\left(\sigma_{i}\right)=\alpha 1+\beta e_{i}
$$


for appropriate constants $\alpha$ and $\beta$. We will elaborate on this representation shortly. Here $J_{\infty}$ denotes the algebra generated by the $e_{i}$ for $i=1,2,3, \ldots$. It seems an amazing coincidence that a representation algebra for the Artin Braid group would appear in a context that seems so far away from this structure. The complex source of Jones' algebra makes this connection seem quite mysterious, and the fact that this same algebra appears in statistical mechanics also seems mysterious. What is the source of this apparent connection of the Artin Braid group with algebras and structures coming from quantum physics?

Remark. In the discussion to follow, we will use the bra and ket notations of Dirac and we will write $<v \mid$ for $v^{t}$, using the notation $v^{t}$ for the transpose of a vector $v$. It is to be understood that in the case of a complex vector space, this is the conjugate transpose, but that in the generalizations that we use (over more general rings) we will simply take the formal transpose without conjugation. Later we will construct real-valued representations of the Temperley-Lieb algebra, and there transpose will be the same as conjugate transpose.

For the purpose of this discussion it will be useful to define a projector to be a linear map $P: V \longrightarrow V$ where $V$ is a vector space or a module over a ring $k$, and $P^{2}$ is a non-zero multiple of $P$. Shall call a projector simple if, in a basis, it takes the form $P=v v^{t}$ where $v$ is a column vector and $v^{t}$ is its transpose. Then $v^{t} v$ is the dot product of $v$ with itself and hence a scalar. Therefore

$$
\begin{aligned}
P^{2}= & P P=v v^{t} v v^{t}=v\left[v^{t} v\right] v^{t} \\
& =\left[v^{t} v\right] v v^{t}=\left[v^{t} v\right] P .
\end{aligned}
$$

Because of the ubiquity of projectors in quantum physics, the physicist P.A.M. Dirac devised a beautiful notation for this situation. Dirac would write $\mid v>$ for $v$ and $<v \mid$ for $v^{t}$. He would write

$$
<v|| w>=<v \mid w>=v^{t} w
$$

for the dot product of two vectors in a given basis.

Then one can write $P$ in Dirac notation by the formula

$$
P=|v><v|
$$

and we have

$$
\begin{aligned}
P^{2}=P P & =|v><v||v><v|=|v><v| v><v \mid \\
= & <v|v>| v><v|=<v| v>P .
\end{aligned}
$$

Now consider the algebra generated by two simple projectors $P=|v><v|$ and $Q=|w><w|$. We have

$$
\begin{aligned}
& P^{2}=<v \mid v>P, \\
& Q^{2}=<w \mid w>Q
\end{aligned}
$$

and

$$
\begin{gathered}
P Q P=|v><v||w><w||v><v| \\
=|v><v| w><w|v><v| \\
=<v|w><w| v>|v><v| \\
=<v|w><w| v>P
\end{gathered}
$$


while

$$
\begin{gathered}
Q P Q=|w><w||v><v||w><w| \\
=|w><w| v><v|w><w| \\
=<w|v><v| w>|w><w| \\
=<w|v><v| w>Q \\
=<v|w><w| v>Q .
\end{gathered}
$$

Thus, with $\lambda=<v|w><w| v>$ we have that

$$
\begin{gathered}
P Q P=\lambda P \\
Q P Q=\lambda Q .
\end{gathered}
$$

We can define $e=P /\langle v| v>$ and $f=Q /<w \mid w>$ and find

$$
\begin{gathered}
e^{2}=e \\
f^{2}=f \\
e f e=\kappa e \\
f e f=\kappa f
\end{gathered}
$$

where $\kappa=\lambda /(\langle v|v><w| w>)$. In this way we see that any two simple projectors generate a Jones algebra of type $J_{2}$. In this sense the appearance of such algebras is quite natural. The relationship with braiding remains as remarkable as ever.

In order to see how these representations work, it is useful to discuss the combinatorics of these algebras a bit further. The Temperley Lieb algebra $T L_{n}[\mathbf{1 1}]$ is an algebra over a commutative ring $k$ with generators $\left\{1, U_{1}, U_{2}, \ldots, U_{n-1}\right\}$ and relations

$$
\begin{gathered}
U_{i}^{2}=\delta U_{i}, \\
U_{i} U_{i \pm 1} U_{i}=U_{i}, \\
U_{i} U_{j}=U_{j} U_{i},|i-j|>1,
\end{gathered}
$$

where $\delta$ is a chosen element of the ring $k$. These equations give the multiplicative structure of the algebra. The algebra is a free module over the ring $k$ with basis the equivalence classes of these products modulo the given relations.

We will make the ground ring specific in the examples to follow. It is clear that the concepts of Temperley Lieb algebra and Jones algebra are interchangeable. Given a Jones algebra $J_{\infty}$, with $e_{i} e_{i \pm 1} e_{i}=\kappa e_{i}$, let $\delta=1 / \sqrt{\kappa}$ (assuming that this square root exists in the ground ring $k$. Then let $U_{i}=\delta e_{i}$ and we find that $U_{i}^{2}=\delta U_{i}$ with

$$
\begin{aligned}
& U_{i} U_{i \pm 1} U_{i}=(1 / \sqrt{\kappa})^{3} e_{i} e_{i \pm 1} e_{i} \\
= & (1 / \sqrt{\kappa})^{3} \kappa e_{i}=(1 / \sqrt{\kappa}) e_{i}=U_{i},
\end{aligned}
$$

converting the Jones algebra to a Temperley Lieb algebra.

It is useful to see the bare bones of the algebra of two projectors. For this purpose, lets write

$$
P=><
$$


and

Then

$$
Q=][
$$

$$
P P=><>=<>><=<>P
$$

and

while

$$
Q Q=][][=[] Q
$$

$$
\begin{gathered}
P Q P=><][><=<][>P \\
Q P Q=][><][=[><] Q=<][>Q .
\end{gathered}
$$

To see how the representation of the braid group is constructed, lets assume that the scalars $<$ ] and [ $>$ are both equal to 1 and that $\delta=<>=$ []. Then $P$ and $Q$ form a two-generator Temperley Lieb algebra $T L_{3}$. We will illustrate how to represent the three strand Artin braid group $B_{3}$ to $T L_{2}$.

It is useful to use the iconic symbol $><$ for a projector and to choose another iconic symbol $\asymp$ for the identity operator in the algebra. With these choices we have

$$
\begin{gathered}
\asymp \asymp=\asymp \\
\asymp><=><\asymp=>< \\
\asymp][=][\asymp=][
\end{gathered}
$$

We define the representation $\rho: B_{3} \longrightarrow T L_{3}$ on the generators $\sigma_{1}=\sigma$ and $\sigma_{2}=\tau$ of the three strand braid group, whose relations are $\sigma \tau \sigma=\tau \sigma \tau$ plus the invertibility of the generators. We define

$$
\begin{gathered}
\rho(\sigma)=A 1+B P=A \asymp+B>< \\
\rho\left(\sigma^{-1}\right)=B 1+A P=B \asymp+A><
\end{gathered}
$$

and

$$
\begin{gathered}
\rho(\tau)=A 1+B Q=A \asymp+B][ \\
\left.\rho\left(\tau^{-1}\right)=B 1+A Q=B \asymp+A\right][.
\end{gathered}
$$

where $A$ and $B$ are commuting indeterminates.

With these definitions, we have

$$
\begin{gathered}
\rho(\sigma)=A \asymp+B>< \\
\rho\left(\sigma^{-1}\right)=B \asymp+A><.
\end{gathered}
$$

Thus

$$
\begin{gathered}
\asymp=(A \asymp+B><)(B \asymp+A><) \\
=A B \asymp \asymp+A^{2} \asymp><+B^{2}><\asymp+A B><>< \\
=A B \asymp+A^{2}><+B^{2}><+A B \delta>< \\
\asymp=A B \asymp+\left(A^{2}+B^{2}+A B \delta\right)><
\end{gathered}
$$

Consequently, we will have $1=\rho(\sigma) \rho\left(\sigma^{-1}\right)$ if we take $B=A^{-1}$ and $\delta=-A^{2}-A^{-2}$. We shall take these values from now on so that

$$
\rho(\sigma)=A \asymp+A^{-1}><=A 1+A^{-1} P
$$


and

$$
\left.\rho(\tau)=A \asymp+A^{-1}\right]\left[=A 1+A^{-1} Q .\right.
$$

With these specializations of $A$ and $B$, it is easy to verify that $\rho$ is a representation of the Artin Braid Group. Note that $P^{2}=\delta P, Q^{2}=\delta Q$ and $P Q P=P$.

$$
\begin{gathered}
\rho(\sigma) \rho(\tau) \rho(\sigma)=\left(A+A^{-1} P\right)\left(A+A^{-1} Q\right)\left(A+A^{-1} P\right) \\
=\left(A^{2}+Q+P+A^{-2} P Q\right)\left(A+A^{-1} P\right) \\
=A^{3}+A Q+A P+A^{-1} P Q+A P+A^{-1} Q P+A^{-1} P^{2}+A^{-3} P Q P \\
=A^{3}+A Q+A P+A^{-1} P Q+A P+A^{-1} Q P+A^{-1} \delta P+A^{-3} P \\
=A^{3}+\left(2 A++A^{-1}\left(-A^{2}-A^{-2}\right)+A^{-3}\right) P+A Q+A^{-1}(P Q+Q P) \\
=A^{3}+A P+A Q+A^{-1}(P Q+Q P) \\
\rho(\sigma) \rho(\tau) \rho(\sigma)=A^{3}+A(P+Q)+A^{-1}(P Q+Q P)
\end{gathered}
$$

Since this last expression is symmetric in $P$ and $Q$, we conclude that

$$
\rho(\sigma) \rho(\tau) \rho(\sigma)=\rho(\tau) \rho(\sigma) \rho(\tau)
$$

Hence $\rho$ is a representation of the Artin Braid Group.

This argument generalizes to yield a corresponding representation of the Artin Braid Group $B_{n}$ to the Temperley Lieb algebra $T L_{n}$ for each $n=2,3, \ldots$. We will discuss the structure of these representations below. In the next section we show how the Jones polynomial can be constructed by a state summation model. This model can be also be viewed as a generalization of the above representation of the Temperley Lieb algebra.

The very close relationship between elementary quantum mechanics and topology is very well illustrated by the structure and representations of the Temperley Lieb algebra. 

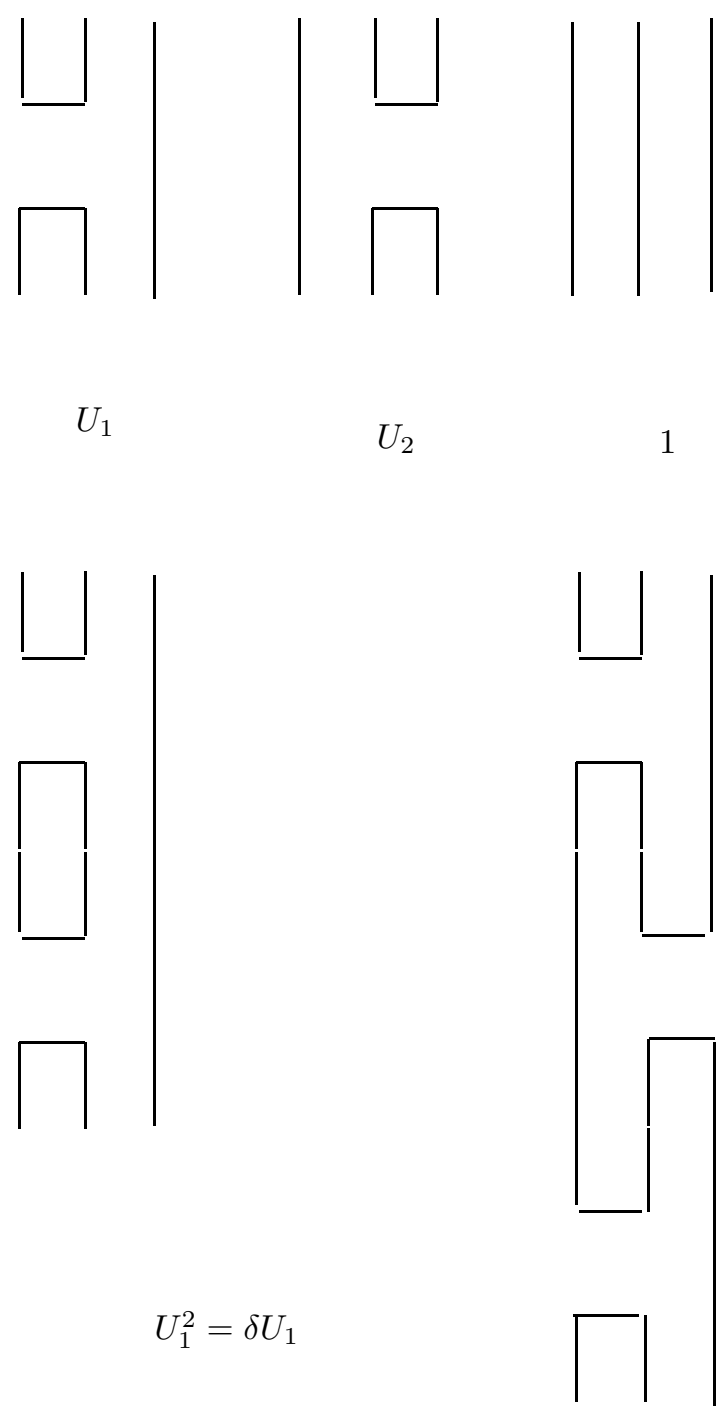

$U_{1} U_{2} U_{1}=U_{1}$

Figure 3 - Diagrammatic Temperley Lieb Algebra

Figure 3 illustrates a diagrammatic interpretation of the Temperley Lieb algebra. In this interpretation, the multiplicative generators of the module are collections of strands connecting $n$ top points and $n$ bottom points. Top points can 
be connected either to top or to bottom points. Bottom points can be connected to either bottom or to top points. All connections are made in the plane with no overlapping lines and no lines going above the top row of points or below the bottom row of points. Multiplication is accomplished by connecting the bottom row of one configuration with the top row of another. In Figure 3 we have illustrated the types of special configurations that correspond to the $U_{i}$, and we have shown that $\delta$ is interpreted as a closed loop.

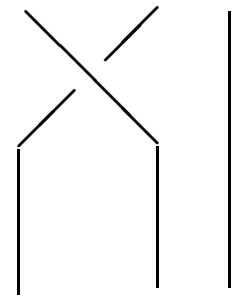

$\sigma_{1}$

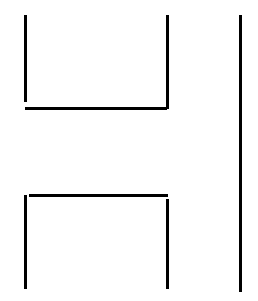

$U_{1}$

$$
\rho\left(\sigma_{1}\right)=A U_{1}+A^{-1} 1
$$

1

Figure 4 - Braid Group Representation 

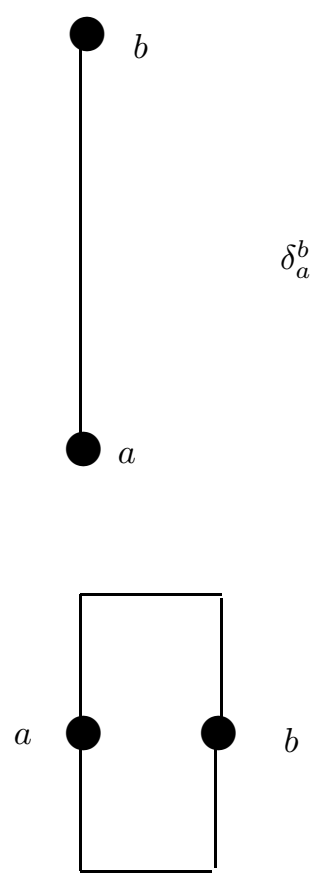

$M^{a b} M_{a b}$

$\delta_{a}^{b}$ $a$

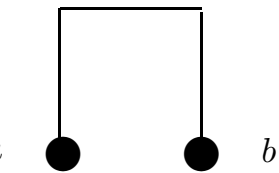

$c$

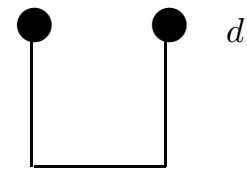

$M^{c d}$
$M_{a b}$

$a$

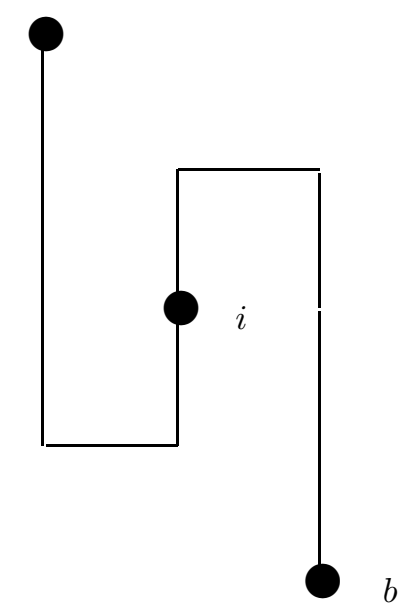

$M^{a i} M_{i b}=\delta_{b}^{a}$

\section{Figure 5 - Abstract Tensors}

One way to make a matrix representation of the Temperley Lieb algebra (and a corresponding representation of the braid group) is to use the matrix $M$ defined as follows

$$
M=\left[\begin{array}{cc}
0 & i A \\
-i A^{-1} & 0
\end{array}\right] .
$$

Note that $M^{2}=1$ where 1 denotes the $(2 \times 2)$ identity matrix. We will use $M$ with either upper or lower indices so that $M^{a b}=M_{a b}$. $M$ will represent both the cup and the cap in the Temperley Lieb diagrams, with $M_{a b}$ representing the cap and $M^{a b}$ representing the cup. If $U$ denotes a cup over a cap, then 
Note that

$$
U_{c d}^{a b}=M^{a b} M_{c d}
$$

Note that

$$
\begin{gathered}
\left(U^{2}\right)_{c d}^{a b}=\Sigma_{i j} U_{i j}^{a b} U_{c d}^{i j}= \\
=\Sigma_{i j} M^{a b} M_{i j} M^{i j} M_{c d}=\left[\Sigma_{i j} M_{i j} M^{i j}\right] M^{a b} M_{c d} \\
=\left[\Sigma_{i j} M_{i j} M^{i j}\right] U_{c d}^{a b} .
\end{gathered}
$$

$$
\Sigma_{i j} M_{i j} M^{i j}=\Sigma_{i j}\left(M_{i j}\right)^{2}=-A^{2}-A^{-2} .
$$

Thus, letting $\delta=-A^{2}-A^{-2}$, we have

$$
U^{2}=\delta U
$$

Then we take $U_{i}$ as a tensor product of identity matrices corresponding to the vertical lines in the diagram for this element and one factor of $U$ for the placement of the cup-cap at the locations $i$ and $i+1$. To see how this works to give the relation $U_{i} U_{i \pm 1} U_{i}=U_{i}$, we verify that $U_{1} U_{2} U_{1}=U_{1}$ in $T L_{3}$. In the calculation to follow we will use the Einstein summation convention. Repeated upper and lower indices are summed across the index set $\{1,2\}$.

$$
U_{1}=U \otimes 1
$$

and

$$
U_{2}=1 \otimes U
$$

so that

and

$$
\left(U_{1}\right)_{d e f}^{a b c}=M^{a b} M_{d e} \delta_{f}^{c}
$$

Therefore

$$
\left(U_{2}\right)_{d e f}^{a b c}=\delta_{d}^{a} M^{b c} M_{e f}
$$

$$
\begin{gathered}
\left(U_{1} U_{2} U_{1}\right)_{d e f}^{a b c}=\left(U_{1}\right)_{i j k}^{a b c}\left(U_{2}\right)_{r s t}^{i j k}\left(U_{1}\right)_{d e f}^{r s t} \\
=\left(M^{a b} M_{i j} \delta_{k}^{c}\right)\left(\delta_{r}^{i} M^{j k} M_{s t}\right)\left(M^{r s} M_{d e} \delta_{f}^{t}\right) \\
=M^{a b}\left(M_{r j} M^{j c}\right)\left(M_{s f} M^{r s}\right) M_{d e}=M^{a b}\left(\delta_{r}^{c}\right)\left(\delta_{f}^{r}\right) M_{d e}= \\
=M^{a b} M_{d e} \delta_{f}^{c}=\left(U_{1}\right)_{d e f}^{a b c}
\end{gathered}
$$

Thus

$$
U_{1} U_{2} U_{1}=U_{1} .
$$

This representation of the Temperley Lieb algebra is useful for knot theory and it is conjectured to be a faithful representation. One may also conjecture that the corresponding braid group representation is faithful. 
Remark. The reader should note that the diagrammatic interpretation of the Temperley Lieb algebra gives a clear way to follow the index details of the calculation we have just performed. In the diagrams an index that is not on a free end is summed over just as in the Einstein summation convention. An index at the end of a line is a free index and does not receive summation. See Figure 5 for an illustration of the index algebra in relation to these diagrams. We will generalize the diagrammatic algebra in section 5 .

3.1. Two Projectors and a Unitary Representation of the Three Strand Braid Group. The Temperley Lieb representation of the braid group that we have described is not a unitary representation except when $A^{2}=-1$, a value that is not of interest in the knot theory. In order to find elementary unitary representations of the braid group, one has to go deeper.

It is useful to think of the Temperley Lieb algebra as generated by projections $e_{i}=U_{i} / \delta$ so that $e_{i}^{2}=e_{i}$ and $e_{i} e_{i \pm 1} e_{i}=\tau e_{i}$ where $\tau=\delta^{-2}$ and $e_{i}$ and $e_{j}$ commute for $|i-j|>1$.

With this in mind, consider elementary projectors $e=|A><A|$ and $f=$ $|B><B|$. We assume that $\left\langle A|A>=<B| B>=1\right.$ so that $e^{2}=e$ and $f^{2}=f$. Now note that

$$
\text { efe }=|A><A| B><B|A><A|=<A|B><B| A>e=\tau e
$$

Thus

$$
e f e=\tau e
$$

where $\tau=<A|B><B| A>$.

This algebra of two projectors is the simplest instance of a representation of the Temperley Lieb algebra. In particular, this means that a representation of the three-strand braid group is naturally associated with the algebra of two projectors, a simple toy model of quantum physics!

Quite specifically if we let $\langle A|=(a, b)$ and $|A\rangle=(a, b)^{t}$ the transpose of this row vector, then

$$
e=|A><A|=\left[\begin{array}{ll}
a^{2} & a b \\
a b & b^{2}
\end{array}\right]
$$

is a standard projector matrix when $a^{2}+b^{2}=1$. To obtain a specific representation, let

$$
e_{1}=\left[\begin{array}{ll}
1 & 0 \\
0 & 0
\end{array}\right]
$$

and

It is easy to check that

$$
e_{2}=\left[\begin{array}{cc}
a^{2} & a b \\
a b & b^{2}
\end{array}\right]
$$

and that

$$
e_{1} e_{2} e_{1}=a^{2} e_{1}
$$




$$
e_{2} e_{1} e_{2}=a^{2} e_{2}
$$

Note also that

$$
e_{1} e_{2}=\left[\begin{array}{cc}
a^{2} & a b \\
0 & 0
\end{array}\right]
$$

and

$$
e_{2} e_{1}=\left[\begin{array}{cc}
a^{2} & 0 \\
a b & 0
\end{array}\right]
$$

We define

$$
U_{i}=\delta e_{i}
$$

for $i=1,2$ with $a^{2}=\delta^{-2}$. Then we have, for $i=1,2$

$$
\begin{gathered}
U_{i}^{2}=\delta U_{i} \\
U_{1} U_{2} U_{1}=U_{1} \\
U_{2} U_{1} U_{2}=U_{2}
\end{gathered}
$$

and

$$
\operatorname{trace}\left(U_{1}\right)=\operatorname{trace}\left(U_{2}\right)=\delta
$$

while

$$
\operatorname{trace}\left(U_{1} U_{2}\right)=\operatorname{trace}\left(U_{2} U_{1}\right)=1 .
$$

We will use these results on the traces of these matrices in Section 6 .

Now we return to the matrix parameters: Since $a^{2}+b^{2}=1$ this means that $\delta^{-2}+b^{2}=1$ whence

$$
b^{2}=1-\delta^{-2}
$$

Therefore $b$ is real when $\delta^{2}$ is greater than or equal to 1 .

We are interested in the case where $\delta=-A^{2}-A^{-2}$ and $A$ is a unit complex number. Under these circumstances the braid group representation

$$
\rho\left(\sigma_{i}\right)=A U_{i}+A^{-1} 1
$$

will be unitary whenever $U_{i}$ is a real symmetric matrix. Thus we will obtain a unitary representation of the three-strand braid group $B_{3}$ when $\delta^{2} \geq 1$. Specifically, let $A=e^{i \theta}$. Then $\delta=-2 \cos (2 \theta)$, so the condition $\delta^{2} \geq 1$ is equivalent to $\cos ^{2}(2 \theta) \geq$ $1 / 4$. Thus we get the specific range of angles $|\theta| \leq \pi / 6$ and $|\theta-\pi| \leq \pi / 6$ that gives unitary representations of the three-strand braid group. 
3.2. Pairs of Projectors and the Alexander Polynomial. Just for the record we note a more general braid group representation that is available via our remarks about the structure of two projectors. Let $\left\{W_{1}, W_{2}, \ldots, W_{n-1}, W_{n}\right\}$ be the standard basis of column vectors for a module of dimension $n$ over $k=C\left[A, A^{-1}\right]$ where $C$ denotes the complex numbers and $W_{k}$ is an $n$-tuple whose entries are zero in all places except the $k$-th place where the entry is one. We shall refer to linear combinations of the $W_{k}$ as vectors over $k$. Given any vector $v$ over $k$, let $\mid v>$ denote $v$ as a column vector, and let $\langle v|=v^{t}$ denote its transpose (just the transpose, as in our previous remarks), the corresponding row vector. Then $P(v)=|v><v|$ is a matrix such that $P^{2}=\langle v \mid v\rangle P$, and $\langle v \mid v\rangle=v^{t} v$ is equal to the sum of the squares of the entries of $v$.

For $k=1,2, \ldots, n-1$ and $i^{2}=-1$, let

and

$$
v_{k}=i A W_{k}-i A^{-1} W_{k+1}
$$

Then, with $\delta=-A^{2}-A^{-2}$,

$$
U_{k}=\left|v_{k}><v_{k}\right| .
$$

$$
\begin{gathered}
U_{k}^{2}=\delta U_{k} \\
U_{k} U_{k \pm 1} U_{k}=U_{k} \\
U_{k} U_{l}=U_{l} U_{k}=0,|k-l|>1 .
\end{gathered}
$$

Thus these matrices give a special representation of the Temperley Lieb algebras $T L_{n}$ for each $n$. Since the loop value is as given above, we can make corresponding representations of the Artin Braid Groups $B_{n}$ by the formulas

$$
\begin{aligned}
& \rho\left(\sigma_{k}\right)=A I_{n}+A^{-1} U_{k}, \\
& \rho\left(\sigma_{k}^{-1}\right)=A^{-1} I_{n}+A U_{k}
\end{aligned}
$$

where $I_{n}$ denotes the $n \times n$ identity matrix. It is not hard to verify that this representation of $B_{n}$ is equivalent to the classical Burau representation (See [15]) of the braid group. This shows that there is a pathway from the algebra of projectors to the Alexander polynomial! We will treat this theme in a separate paper.

\section{The Bracket Polynomial}

In this section we shall discuss the structure of the bracket state model for the Jones polynomial [11]. In this way, we will explicitly construct the Jones polynomial by using a state summation that is closely related to the braid group representation described in the last section.

Before discussing the bracket polynomial we recall the basic theorem of Reidemeister [23] about knot and link diagrams. Reidemeister proved that the three local moves on diagrams illustrated in Figure 6 capture combinatorially the notion of ambient isotopy of links and knots in three-dimensional space. That is, if two diagrams represent knots or links that are isotopic in three-dimensional space, then the one diagram can be obtained from the other by a sequence of Reidemeister moves. It is understood that a Reidemeister move is a local change on the diagram 
and that it is locally just as indicated by the picture of the move. That is, a type one move adds or eliminates a loop in the underlying 4-regular graph of the knot diagram. A type two move operates on a two sided region and a type three move operates on a three sided region. It is also understood that one can simplify a diagram by a homeomorphism of the plane. This could be called the type zero move, but it is always available. The equivalence relation generated by the type two and type three moves is called regular isotopy. The bracket polynomial is a regular isotopy invariant that can be normalized to produce an invariant of all three Reidemeister moves.
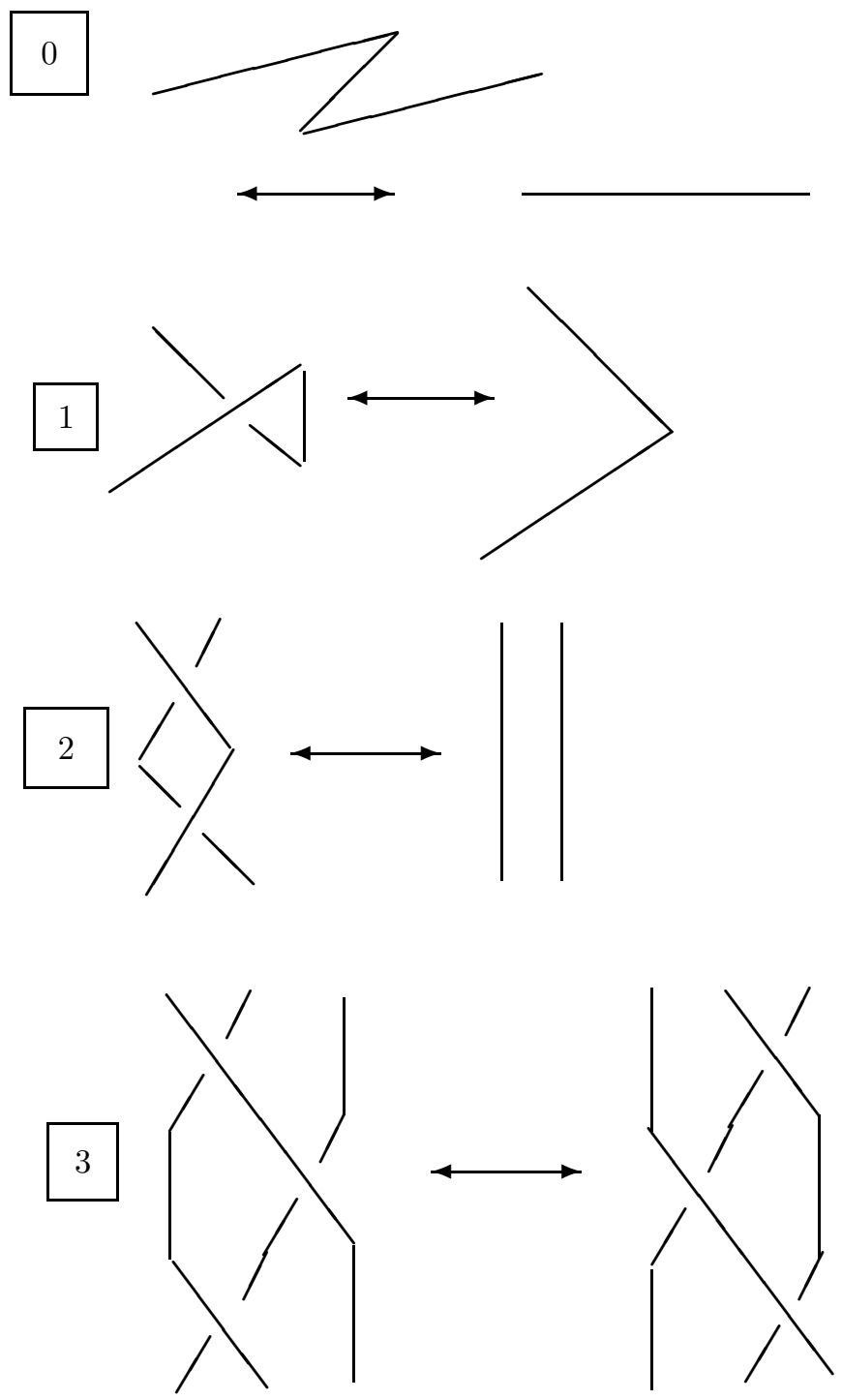

Figure 6 - The Reidemeister Moves

The bracket polynomial , $\langle K\rangle=\langle K\rangle(A)$, assigns to each unoriented link diagram $K$ a Laurent polynomial in the variable $A$ such that 
(1) If $K$ and $K^{\prime}$ are regularly isotopic links, then $\langle K\rangle=\left\langle K^{\prime}\right\rangle$.

(2) If $K O$ denotes the disjoint union of $K$ with an extra unknotted and unlinked component $O$, then

$$
<K \quad O>=\delta<K>
$$

where

$$
\delta=-A^{2}-A^{-2} .
$$

(3) $<K>$ satisfies the following formula where in Figure 7 the small diagrams represent parts of larger diagrams that are identical except at the site indicated in the bracket. In the text formula we have used the notations $S_{A} K$ and $S_{B} K$ to indicate the two smoothings of a single crossing in the diagram $K$. That is, $K, S_{A} K$ and $S_{B} K$ differ at the site of one crossing in the diagram $K$. The convention for these smoothings is indicated in Figure 7 .

$$
<K>=A<S_{A} K>+A^{-1}<S_{B} K>
$$

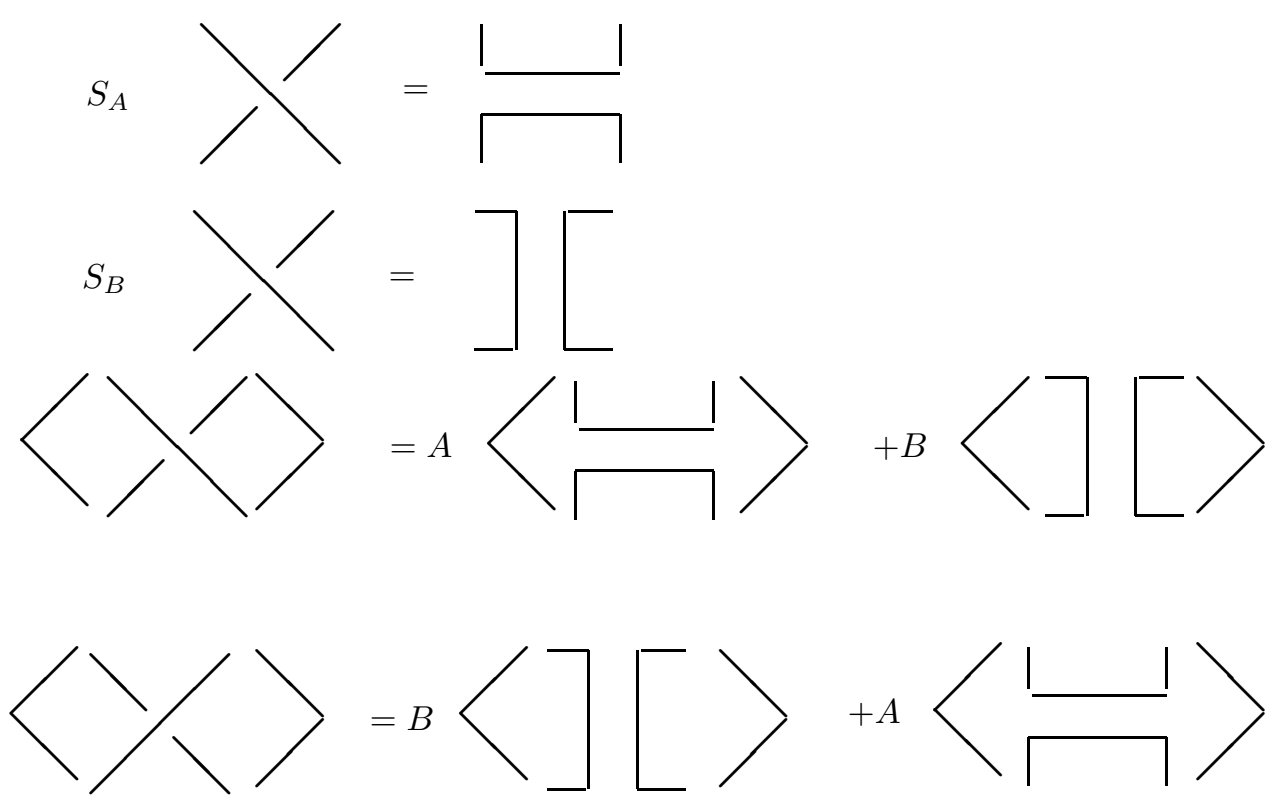

Figure 7 - Bracket Expansion

This formula for expanding the bracket polynomial can be indicated symbolically in the same fashion that we used in the previous section to indicate the representation of the Artin Braid Group to the Temperley Lieb algebra. We will denote a crossing in the link diagram by the letter chi, $\chi$. The letter itself denotes a crossing where the curved line in the letter chi is crossing over the straight segment in the letter. The barred letter denotes the switch of this crossing where the curved line in the letter chi is undercrossing the straight segment in the letter. In the state model a crossing in a diagram for the knot or link is expanded into two possible states by either smoothing (reconnecting) the crossing horizontally, $\asymp$, or vertically $><$. Coefficients in this expansion correspond exactly to our representation of the 
braid group so that any closed loop (without crossings) in the plane has value $\delta=-A^{2}-A^{-2}$ and the crossings expand according to the formulas

$$
\begin{gathered}
\chi=A \asymp+A^{-1}>< \\
\bar{\chi}=A^{-1} \asymp+A><.
\end{gathered}
$$

The verification that the bracket is invariant under the second Reidemeister move is then identical to our proof in the previous section that

$$
\chi \bar{\chi}=\asymp .
$$

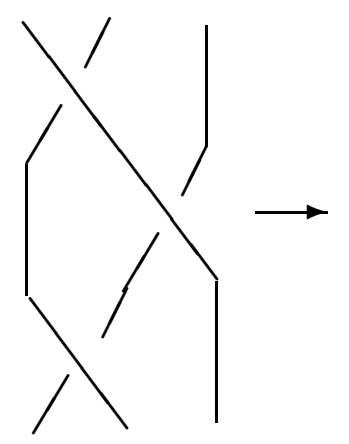

$A$
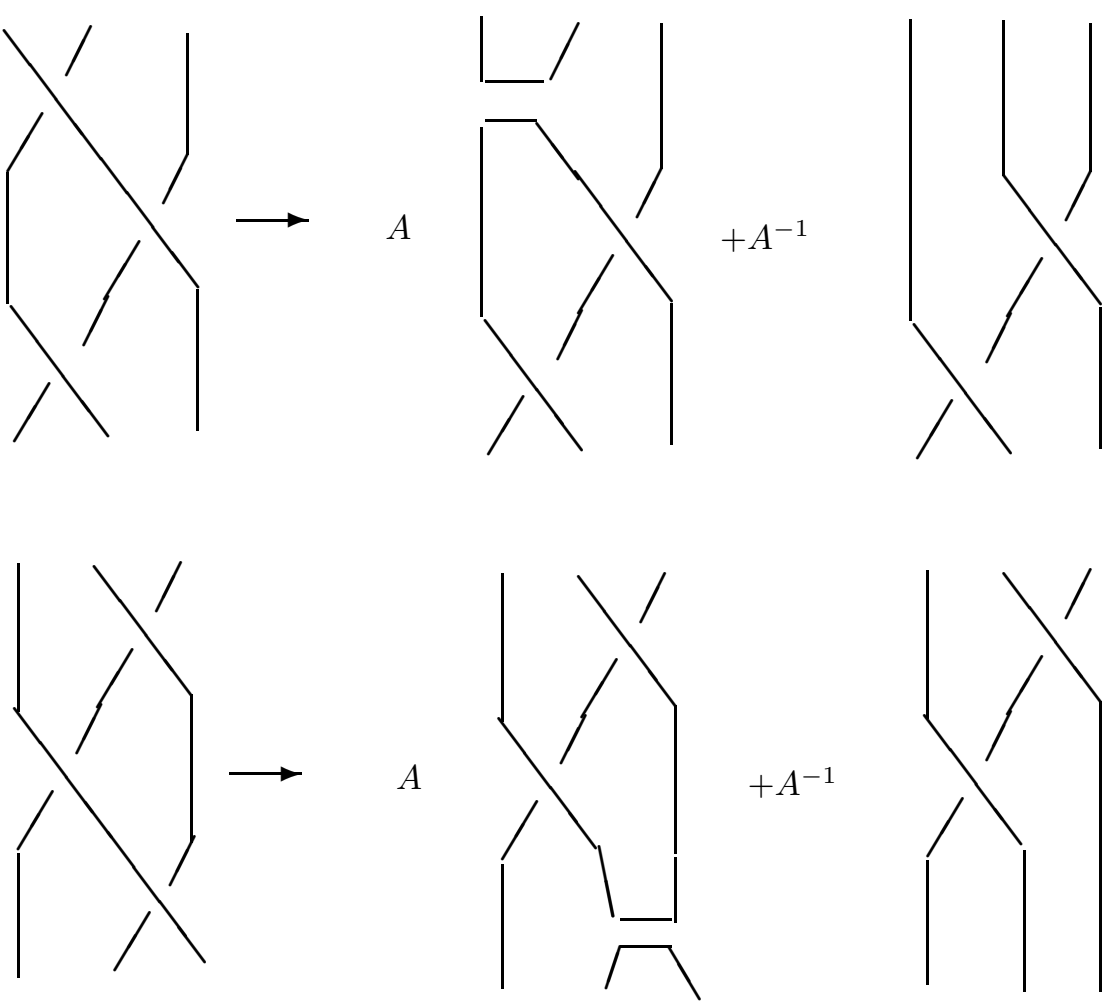

Figure 8 - Invariance of Bracket under Third Reidemeister Move 


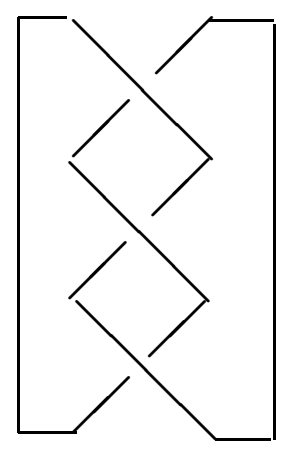

$K$
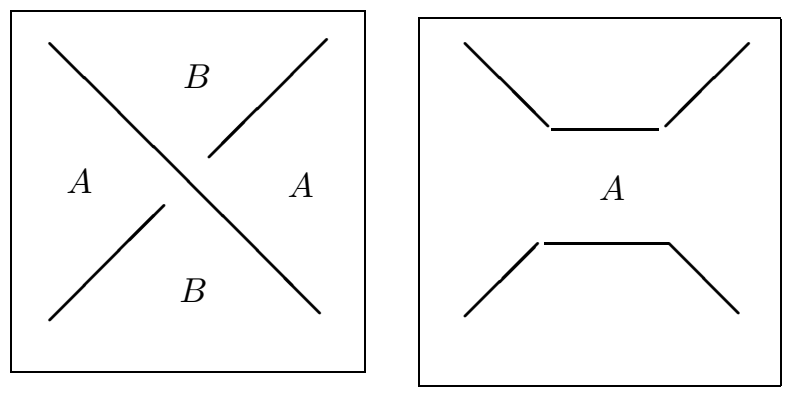

$$
<K>=\Sigma_{S}<K \mid S>d^{\|S\|}
$$

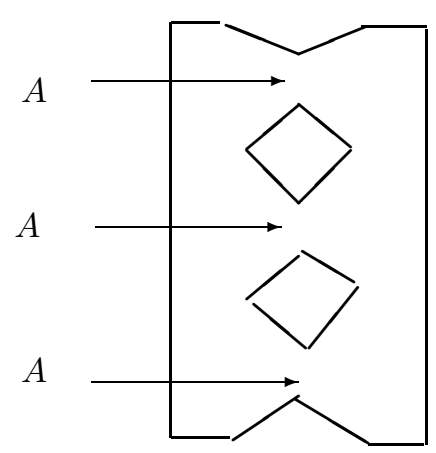

$<K \mid S>=A^{3}$

$$
\|S\|=3
$$

$S$

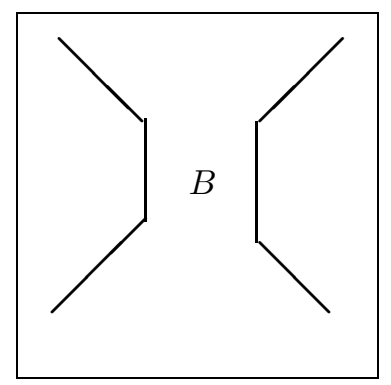

Figure 9 - Bracket States

Knowing that the bracket is invariant under the second Reidemeister move allows us to verify directly that it is invariant under the third Reidemeister move. This is illustrated in Figure 8. In this Figure we show the two equivalent configurations in the third Reidemeister move vertically on the left, with arrows point to the right of each configuration to an expansion via the bracket at one crossing. The expansions give the same bracket calculation due to invariance under the second Reidemeister move. Since the bracket is invariant under the second and third Reidemeister moves, property 1. is a direct consequence of properties 2. and 3.. The second two properties define the bracket on arbitrary link diagrams.

In fact we could have begun with the following more general definition: Let $K$ be any unoriented link diagram. Define a state of $K$ to be a choice of smoothings for all the crossings of $K$. There are $2^{N}$ states of a diagram with $N$ crossings. A smoothing of a crossing is a local replacement of that crossing with two arcs that do not cross one another, as shown below. There are two choices for smoothing a given crossing. In illustrating a state it is convenient to label the smoothing with 
$A$ or $B$ to indicate the crossing from which it was smoothed. The $A$ or $B$ is called a vertex weight of the state.

Label each state with vertex weights $A$ or $B$ as illustrated in Figure 9. Here $A$ and $B$ are commuting polynomial variables. Define two evaluations related to the state: The first evaluation is the product of the vertex weights, denoted

$$
[K \mid S] \text {. }
$$

The second evaluation is the number of loops (Jordan curves) in the state $S$, denoted

$$
\|S\| \text {. }
$$

Define the state summation, $[K]$, by the formula

$$
[K]=\sum_{S}[K \mid S] \delta^{\|S\|-1} .
$$

It follows from this definition,that $[K]$ satisfies the formulas

$$
\begin{gathered}
{[\chi]=A[\asymp]+B[><]} \\
{[O \quad K]=\delta[K],}
\end{gathered}
$$

and

$$
[O]=1 \text {. }
$$

The demand that $[K]$ be invariant under the second Reidemeister move leads to the conditions $B=A^{-1}$ and $\delta=-A^{2}+A^{-2}$. This specialization is easily seen to be invariant under the third Reidemeister move. Calling this specialization the topological bracket, and denoting it (as above) by $\langle K\rangle$ one finds the following behavior under the first Reidemeister move

$$
<\gamma>=-A^{3}<\smile>
$$

and

$$
<\bar{\gamma}>=-A^{-3}<\smile>
$$

where $\gamma$ denotes a curl of positive type as indicated in Figure 10, and $\bar{\gamma}$ indicates a curl of negative type as also seen in this Figure.

The topological bracket is invariant under regular isotopy and can be normalized to an invariant of ambient isotopy by the definition

$$
f_{K}(A)=\left(-A^{3}\right)^{-w(K)}<K>(A)
$$

where $w(K)$ is the sum of the crossing signs of the oriented link $K . w(K)$ is called the writhe of $K$. The convention for crossing signs is shown in Figure 10.
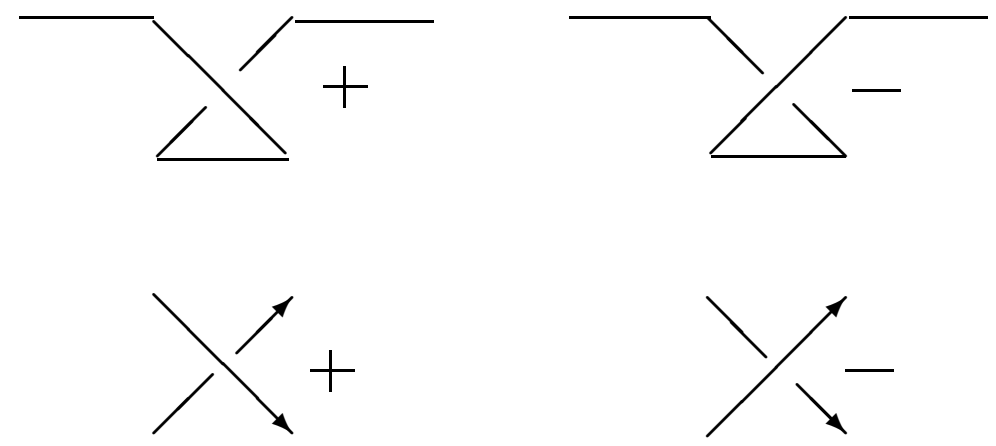


\section{Figure 10 - Crossing Signs}

By a change of variables one obtains the original Jones polynomial, $V_{K}(t)[\mathbf{1 0}]$ from the normalized bracket:

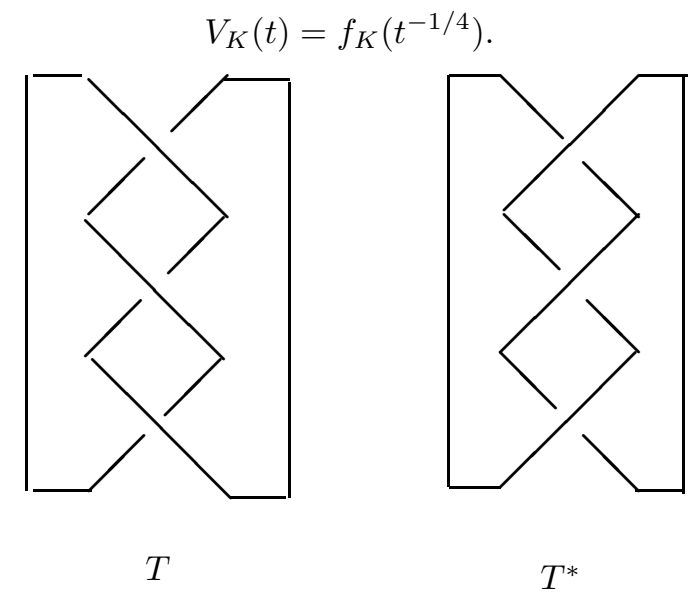

Figure 11 - Trefoil and Mirror Image

The bracket model for the Jones polynomial is quite useful both theoretically and in terms of practical computations. One of the neatest applications is to simply compute $f_{K}(A)$ for the trefoil knot $T$ and determine that $f_{K}(A)$ is not equal to $f_{K}\left(A^{-1}\right)$. This shows that the trefoil is not ambient isotopic to its mirror image (See Figure 11), a fact that is quite tricky to prove by classical methods.

Remark. The relationship of the Temperley Lieb algebra with the bracket polynomial comes through the basic bracket identity. This identity, interpreted in the context of the diagrammatic Temperley Lieb algebra becomes a representation $\rho$ of the Artin braid group $B_{n}$ on $n$ strands to the Temperley Lieb algebra $T L_{n}$ defined by the formulas

$$
\begin{gathered}
\rho\left(\sigma_{i}\right)=A U_{i}+A^{-1} 1 \\
\rho\left(\sigma_{i}^{-1}\right)=A^{-1} U_{i}+A 1 .
\end{gathered}
$$

Here sigma $_{i}$ denotes the braid generator that twists strands $i$ and $i+1$. For this representation of the Temperley Lieb algebra, the loop value $\delta$ is $-A^{2}-A^{-2}$ and the ring $k$ is $Z\left[A, A^{-1}\right]$, the ring of Laurent polynomials in $A$ with integer coefficients.

Remark. There are hints of quantum mechanical interpretations in the combinatorics of this state sum model for the Jones polynomial. The expansion formula for the bracket polynomial

$$
<K>=A<S_{A} K>+A^{-1}<S_{B} K>
$$

suggests that the diagram $K$ should be thought of as a superposition of the diagrams $S_{A} K$ and $S_{B} K$. That is, we can think of a knot diagram with respect to 
a given crossing as the superposition of the diagrams obtained by smoothing that crossing. Then, with respect to all the crossings, one can think of the diagram as a superposition of the states obtained by smoothing each crossing in one of its two possible ways. This is a superposition view of the bracket state sum as a whole.

$$
<K>=\sum_{S}<K \mid S>\delta^{|| S||-1}
$$

In this sense the bracket polynomial evaluation is directly analogous to an amplitude in quantum mechanics. We shall make this analogy more precise in the sections to follow. However, the topological information is contained in this amplitude as whole, and not in any specific state evaluation. Thus the topological model ignores the standard measurement situation in quantum mechanics where one gets at best information about one state at a time when a measurement is taken. This means that a quantum computational model of the bracket polynomial will be essentially probabilistic, only giving partial information at each measurement.

As a result of this discussion, it is natural to ask to what extent one can extract partial topological information from an incomplete summation over the states of the bracket polynomial. It is not clear at this stage what this answer is to this question. It may require a new exploration of the properties of the state sum and its corresponding polynomial.

\section{Knot Amplitudes}

At the end of the first section we said: the connection of quantum mechanics with topology is an amplification of Dirac notation. In this section we begin the process of amplification!

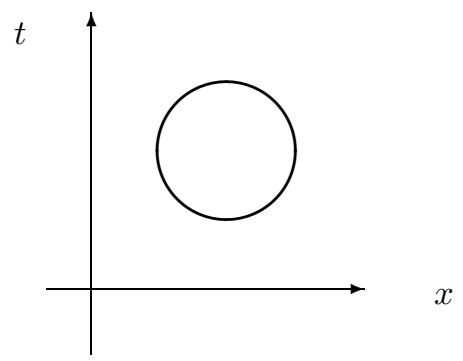

Figure 12 - Circle in Spacetime

Consider first a circle in a spacetime plane with time represented vertically and space horizontally. The circle represents a vacuum to vacuum process that includes the creation of two "particles", and their subsequent annihilation. See Figures 12 and 13 . 


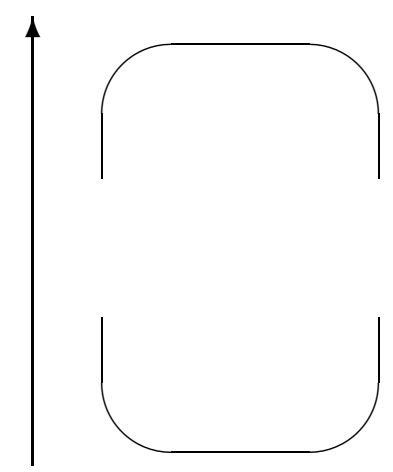

\section{Figure 13 - Creation and Annihilation}

In accord with our previous description, we could divide the circle into these two parts (creation(a) and annihilation (b)) and consider the amplitude $\langle b \mid a\rangle$. Since the diagram for the creation of the two particles ends in two separate points, it is natural to take a vector space of the form $V \otimes V$ as the target for the bra and as the domain of the ket.

We imagine at least one particle property being catalogued by each dimension of $V$. For example, a basis of $V$ could enumerate the spins of the created particles. If $\left\{e_{a}\right\}$ is a basis for $V$ then $\left\{e_{a} \otimes e_{b}\right\}$ forms a basis for $V \otimes V$. The elements of this new basis constitute all possible combinations of the particle properties. Since such combinations are multiplicative, the tensor product is the appropriate construction.

In this language the creation ket is a map cup,

$$
\text { cup }=\mid a>: C \longrightarrow V \otimes V,
$$

and the annihilation bra is a mapping cap,

$$
c a p=<b \mid: V \otimes V \longrightarrow C .
$$

The first hint of topology comes when we realize that it is possible to draw a much more complicated simple closed curve in the plane that is nevertheless decomposed with respect to the vertical direction into many cups and caps. In fact, any simple (no self-intersections) differentiable curve can be rigidly rotated until it is in general position with respect to the vertical. It will then be seen to be decomposed into these minima and maxima. Our prescriptions for amplitudes suggest that we regard any such curve as an amplitude via its description as a mapping from $C$ to $C$.

Each simple closed curve gives rise to an amplitude, but any simple closed curve in the plane is isotopic to a circle, by the Jordan Curve Theorem. If these are topological amplitudes, then they should all be equal to the original amplitude for the circle. Thus the question: What condition on creation and annihilation will insure topological amplitudes? The answer derives from the fact that all isotopies of the simple closed curves are generated by the cancellation of adjacent maxima and minima as illustrated below. 


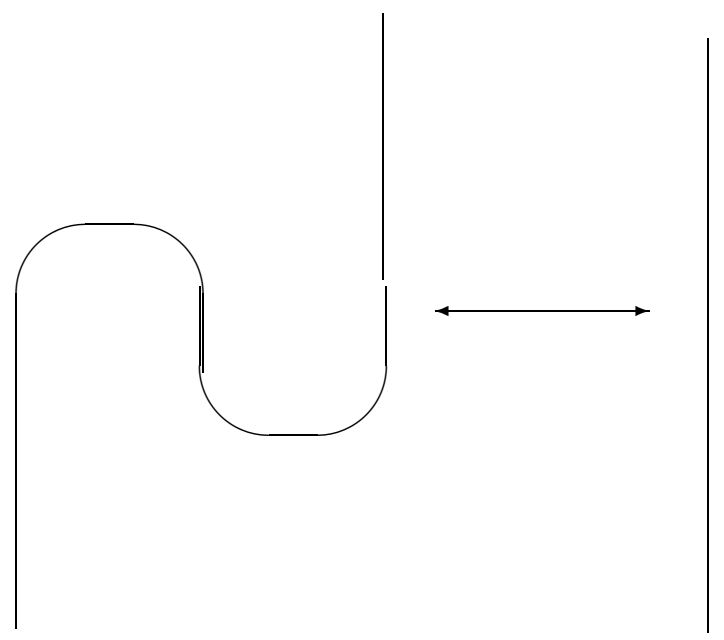

Figure 14 - Cancellation of Maxima and Minima

In composing mappings it is necessary to use the identifications $(V \otimes V) \otimes V=$ $V \otimes(V \otimes V)$ and $V \otimes k=k \otimes V=V$. Thus in the illustration above, the composition on the left is given by

$$
\begin{gathered}
V=V \otimes k-1 \otimes \text { cup } \rightarrow V \otimes(V \otimes V) \\
=(V \otimes V) \otimes V-c a p \otimes 1 \rightarrow k \otimes V=V .
\end{gathered}
$$

This composition must equal the identity map on $V$ (denoted 1 here) for the amplitudes to have a proper image of the topological cancellation. This condition is said very simply by taking a matrix representation for the corresponding operators.

Specifically, let $\left\{e_{1}, e_{2}, \ldots, e_{n}\right\}$ be a basis for $V$. Let $e_{a b}=e_{a} \otimes e_{b}$ denote the elements of the tensor basis for $V \otimes V$. Then there are matrices $M_{a b}$ and $M^{a b}$ such that

$$
\operatorname{cup}(1)=\Sigma M^{a b} e_{a b}
$$

with the summation taken over all values of $a$ and $b$ from 1 to $n$. Similarly, cap is described by

$$
\operatorname{cap}\left(e_{a b}\right)=M_{a b .}
$$

Thus the amplitude for the circle is

$$
\operatorname{cap}[\operatorname{cup}(1)]=\operatorname{cap} \Sigma M^{a b} e_{a b}=\Sigma M^{a b} M_{a b} .
$$

In general, the value of the amplitude on a simple closed curve is obtained by translating it into an "abstract tensor expression" in the $M_{a b}$ and $M^{a b}$, and then summing over these products for all cases of repeated indices.

Returning to the topological conditions we see that they are just that the matrices $\left(M_{a b}\right)$ and $\left(M^{a b}\right)$ are inverses in the sense that $\Sigma M_{a i} M^{i b}=\delta_{a}^{b}$ and $\Sigma M^{a i} M_{i b}=\delta_{b}^{a}$ where $\delta_{a}^{b}$ denotes the (identity matrix) Kronecker delta that is equal to one when its two indices are equal to one another and zero otherwise. 


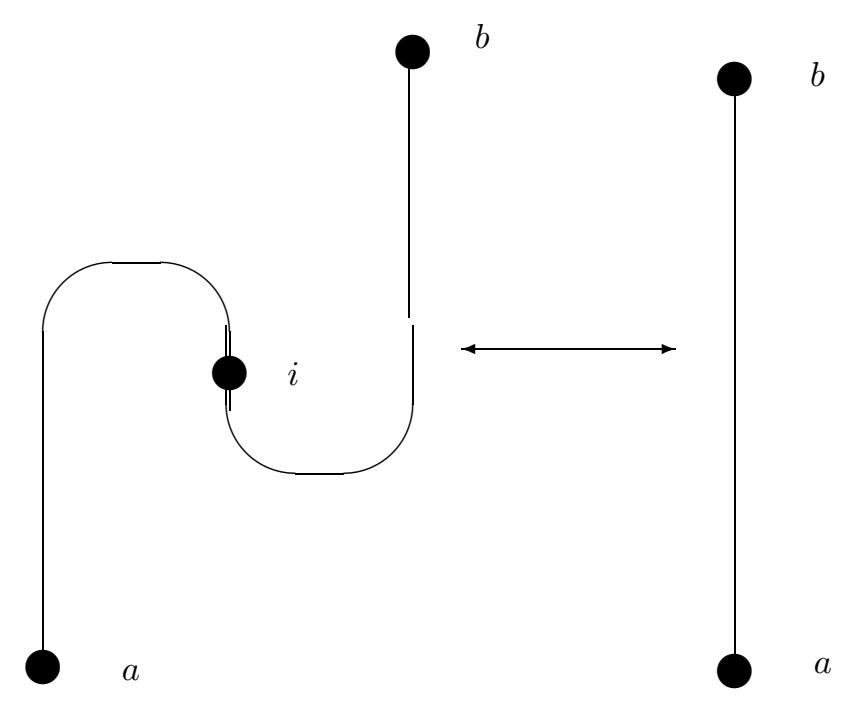

Figure 15 - Algebraic Cancellation of Maxima and Minima $\delta_{a}^{b}$.

In Figure 15, we show the diagrammatic representative of the equation $\Sigma M_{a i} M^{i b}=$

In the simplest case cup and cap are represented by $2 \times 2$ matrices. The topological condition implies that these matrices are inverses of each other. Thus the problem of the existence of topological amplitudes is very easily solved for simple closed curves in the plane.

Now we go to knots and links. Any knot or link can be represented by a picture that is configured with respect to a vertical direction in the plane. The picture will decompose into minima (creations) maxima (annihilations) and crossings of the two types shown below. (Here I consider knots and links that are unoriented. They do not have an intrinsic preferred direction of travel.) See Figure 16. In Figure 16 we have indicated the crossings as mappings of $V \otimes V$ to itself , called $R$ and $R^{-1}$ respectively. These mappings represent the transitions corresponding to these elementary configurations. 


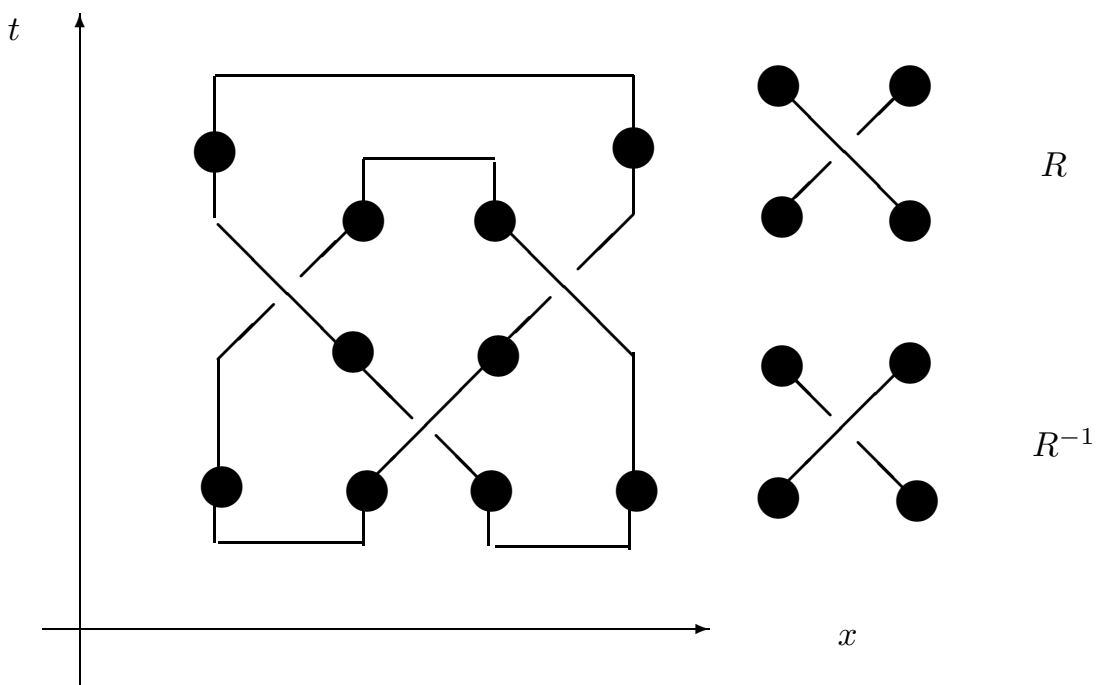

Figure 16 - Morse Knot Decomposition

That $R$ and $R^{-1}$ really must be inverses follows from the isotopy shown in Figure 17 (This is the second Reidemeister move.)

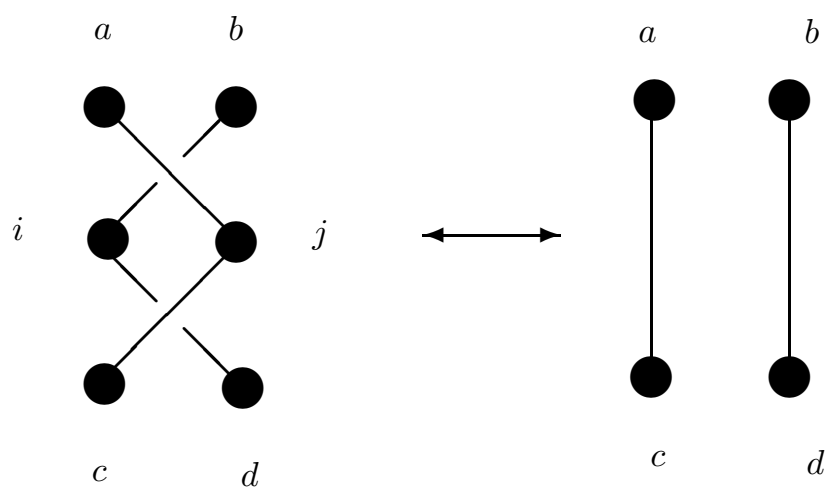

Figure 17 - Braiding Cancellation

We now have the vocabulary of cup,cap, $R$ and $R^{-1}$. Any knot or link can be written as a composition of these fragments, and consequently a choice of such mappings determines an amplitude for knots and links. In order for such an amplitude to be topological we want it to be invariant under the list of local moves on the diagrams shown in Figure 18. These moves are an augmented list of the Reidemeister moves, adjusted to take care of the fact that the diagrams are arranged with respect to a given direction in the plane. The equivalence relation generated by these moves is called regular isotopy. It is one move short of the relation known as 
ambient isotopy. The missing move is the first Reidemeister move shown in Figure 6.

In the first Reidemeister move, a curl in the diagram is created or destroyed. Ambient isotopy (generated by all the Reidemeister moves) corresponds to the full topology of knots and links embedded in three dimensional space. Two link diagrams are ambient isotopic via the Reidemeister moves if and only if there is a continuous family of embeddings in three dimensions leading from one link to the other. The moves give us a combinatorial reformulation of the spatial topology of knots and links.
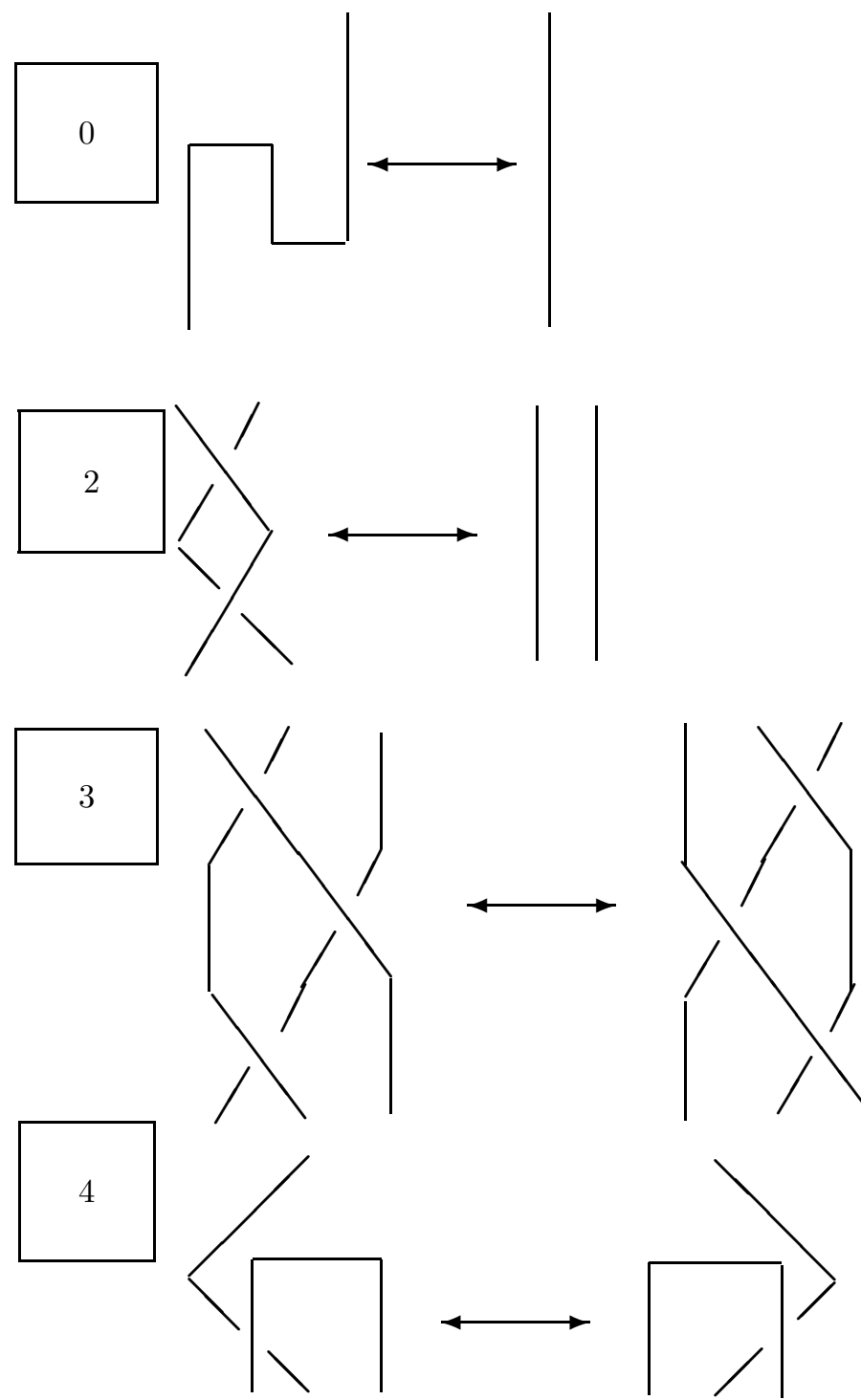

Figure 18- Moves for Regular Isotopy of Morse Diagrams 
By ignoring the first Reidemeister move, we allow the possibility that these diagrams can model framed links, that is links with a normal vector field or,equivalently, embeddings of curves that are thickened into bands. It turns out to be fruitful to study invariants of regular isotopy. In fact, one can usually normalize an invariant of regular isotopy to obtain an invariant of ambient isotopy. We shall see an example of this phenomenon with the bracket polynomial in a few paragraphs.

As the reader can see, we have already discussed the algebraic meaning of moves 0 . and 2. The other moves translate into very interesting algebra. Move 3., when translated into algebra, is the famous Yang-Baxter equation. The Yang-Baxter equation occurred for the first time in problems related to exactly solved models in statistical mechanics (See [19].). All the moves taken together are directly related to the axioms for a quasi-triangular Hopf algebra (aka quantum group). We shall not go into this connection here.

There is an intimate connection between knot invariants and the structure of generalized amplitudes, as we have described them in terms of vector space mappings associated with link diagrams. This strategy for the construction of invariants is directly motivated by the concept of an amplitude in quantum mechanics. It turns out that the invariants that can actually be produced by this means (that is by assigning finite dimensional matrices to the caps, cups and crossings) are incredibly rich. They encompass, at present, all of the known invariants of polynomial type (Alexander polynomial, Jones polynomial and their generalizations.).

It is now possible to indicate the construction of the Jones polynomial via the bracket polynomial as an amplitude, by specifying its matrices. The cups and the caps are defined by $\left(M_{a b}\right)=\left(M^{a b}\right)=M$ where $M$ is the $2 \times 2$ matrix (with $i i=-1)$.

$$
M=\left[\begin{array}{cc}
0 & i A \\
-i A^{-1} & 0
\end{array}\right]
$$

Note that $M M=I$ where $I$ is the identity matrix. Note also that the amplitude for the circle is

$$
\begin{aligned}
& \Sigma M_{a b} M^{a b}=\Sigma M_{a b} M_{a b}=\Sigma M_{a b}^{2} \\
= & (i A)^{2}+\left(-i A^{-1}\right)^{2}=-A^{2}-A^{-2 .}
\end{aligned}
$$

The matrix $R$ is then defined by the equation

$$
R_{c d}^{a b}=A M^{a b} M_{c d}+A^{-1} \delta_{c}^{a} \delta_{d}^{b}
$$

Since, diagrammatically, we identify $R$ with a (right handed) crossing, this equation can be written diagrammatically as the generating identity for the bracket polynomial:

$$
\chi=A \asymp+A^{-1}><
$$



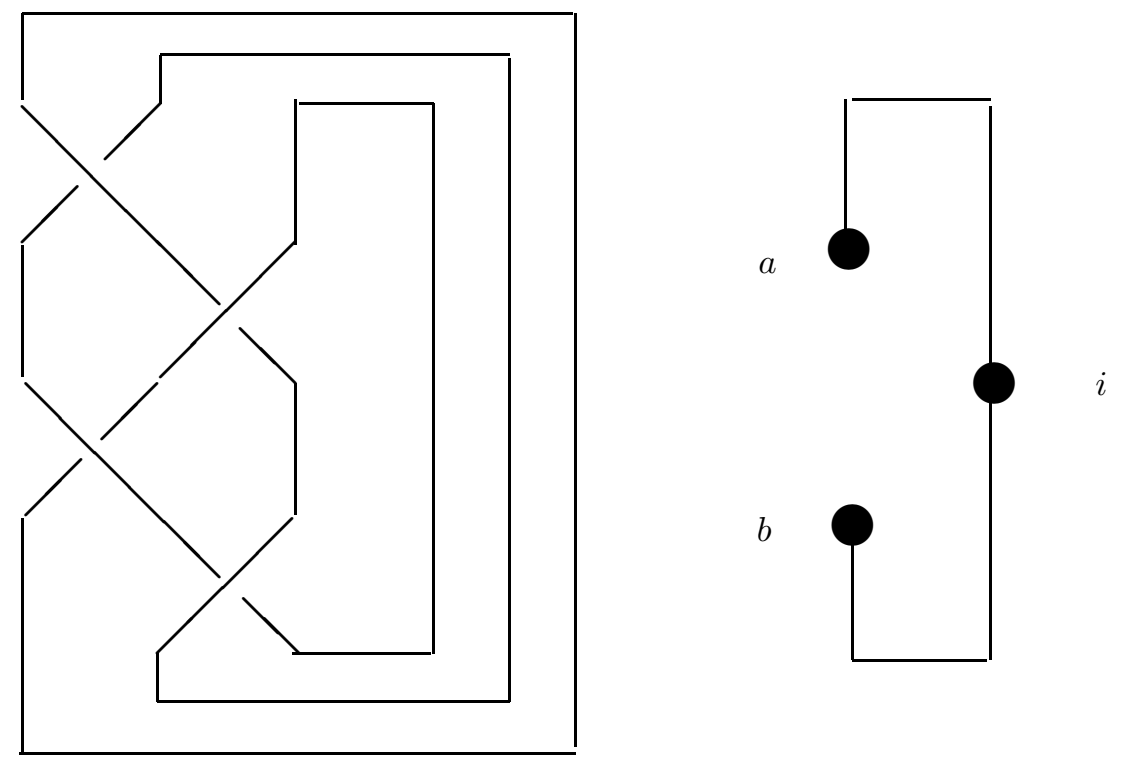

$$
\eta_{a}^{b}=M_{a i} M^{b i}
$$

Figure 19 - Pairing Maxima and Minima for Braid Closures

Taken together with the loop value of $-A^{2}-A^{-2}$ that is a consequence of this matrix choice, these equations can be regarded as a recursive algorithm for computing the amplitude. This algorithm is the bracket state model for the (unnormalized) Jones polynomial [11]. We have discussed this model in the previous sections.

The upshot of these remarks is that the bracket state summation can be reformulated as a matrix model as described in this section. Thus the values of the bracket polynomial can be regarded as generalized quantum amplitudes. Note also that the model that we have described in this section can be seen as a generalization of the representation of the Temperley Lieb algebra from Section 3 with basic matrix $M$ as above. In fact, in the case where the knot or link is a closure of a braid, we can say even more. Suppose that $K=\bar{b}$ where $b$ is an $n$-strand braid in $B_{n}$. Then the cups and caps can be paired off as shown in Figure 19 so that

Here

$$
Z(K)=\delta<K>=\operatorname{Trace}\left(\eta^{\otimes n} \rho(b)\right) .
$$

$$
\eta_{b}^{a}=\Sigma_{i} M_{b i} M^{a i}
$$

so that $\eta=M M^{t}$ where $M^{t}$ denotes the transpose of the matrix $M$.

and $\rho: B_{n} \longrightarrow T L_{n}$ is the matrix representation of the Temperley Lieb algebra specified in Section 3. The key to the workings of this representation of the bracket 
calculation for braids is the fact that for any pure connection element $Q$ (obtained from a product of the $U_{i}^{\prime} s$ ) in the Temperley-Lieb algebra $T L_{n}$, the evaluation

$$
\operatorname{Trace}\left(\eta^{\otimes n} Q\right)
$$

is equal to $\delta^{\lambda(Q)}$ where $\lambda(Q)$ is the number of loops in the (braid) closure of the diagram for $Q$.

In general, if we have a linear function $T R: T L_{n} \longrightarrow Z[\delta]$ such that $T R(Q)=$ $\delta^{\lambda(Q)}$ for elements $Q$ as above, then $T R(\rho(b))=\delta<\bar{b}>$ for any $n$-strand braid $b$.

\section{Quantum Computing}

In this paper I have concentrated on giving a picture of the general framework of the Jones polynomial and how it is related to a very general, in fact categorical, view of quantum mechanics. Many algorithms in quantum topology are configured without regard to unitary evolution of the amplitude since the constraint has been topological invariance rather than conformation to physical reality. This gives rise to a host of problems of attempting to reformulate topological amplitudes as quantum computations. A particular case in point is the bracket model for the Jones polynomial. It would be of great interest to see a reformulation of this algorithm that would make it a quantum computation in the strict sense of quantum computing. One way to think about this is to view the bracket model as a vacuum-vacuum amplitude as we have done in the last section of this paper. Then it can be configured as a composition of operators (cups, caps and braiding). If the braiding is unitary. Then at least this part can be viewed as a quantum computation.

To see how this can be formulated consider the vacuum-vacuum computation of a link amplitude as we have described it in section 4 . In Figure 20 we have indicated an amplitude where the temporal decomposition consists first in a composition of cups (creations), then braiding and then caps (annihilations). Thus we can write the amplitude in the form

$$
Z_{K}=<C U P|M| C A P>
$$




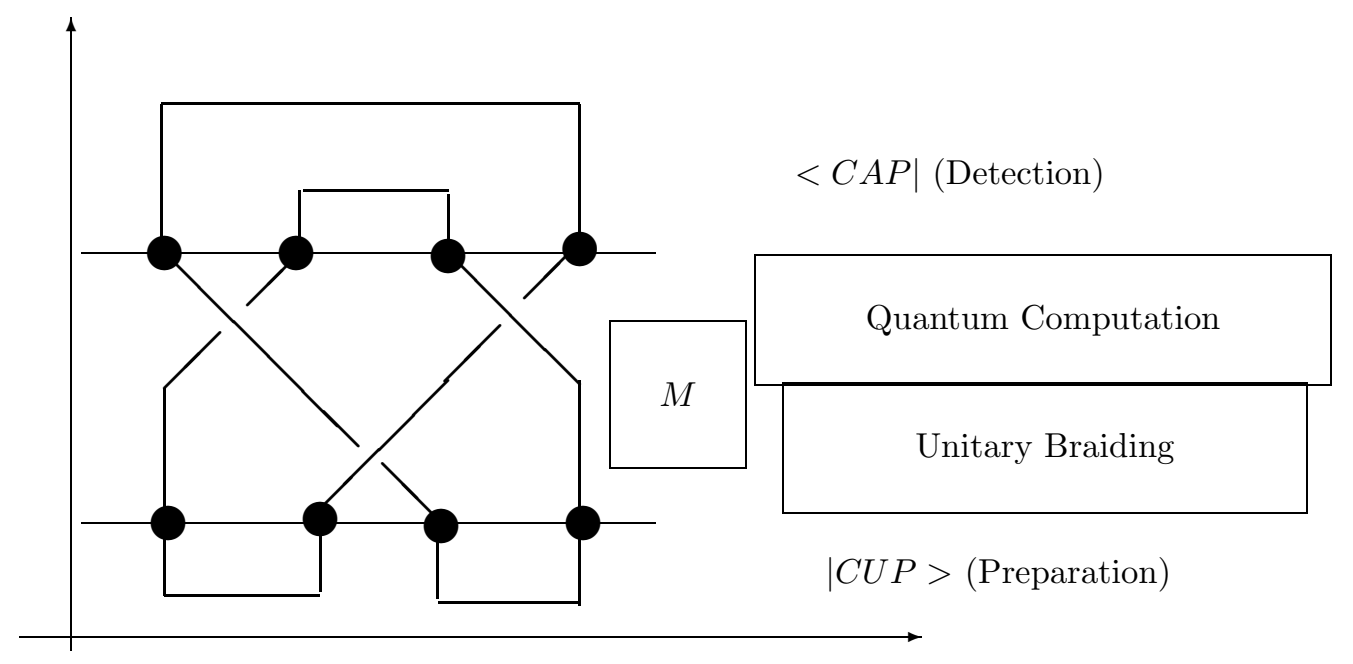

$$
Z_{K}=<C A P|M| C U P>
$$

Figure $20-Z_{K}=<C U P|M| C A P>$ - A Knot Quantum Computer

where $<C U P \mid$ denotes the composition of cups, $M$ is the composition of elementary braiding matrices and $\mid C A P>$ is the composition of caps. We then regard $<C U P \mid$ as the preparation of this state and $\mid C A P>$ as the detection of this state. In order to view $Z_{K}$ as a quantum computation, we need that $M$ be a unitary operator. This will be the case if the $R$-matrices (the solutions to the Yang-Baxter equation used in the model for this amplitude) are unitary. In this case, each $R$-matrix can be viewed as a quantum gate (or possibly a composition of quantum gates) and the vacuum-vacuum diagram for the knot is interpreted as a quantum computer. This quantum computer will probabalistically compute the values of the states in the state sum for $Z_{K}$. In order to do so, we would need to specify those observations and preparations that correspond to the cups and the caps in the diagram. A more modest proposal is to regard the braiding sector of the diagram as a quantum computer. That braiding sector will represent a unitary evolution, and one can ask more generally what can be computed by using such a gate derived from a braid.

It should be noted that because the quantum computer gives probabilistic data, it cannot compute the knot invariants exactly. In fact, the situation is more serious than that. If we assume that the parameters in the knot invariant are complex, then the computer will only find (probabilistically) the absolute squares of the various complex parameters. Important phase information will be lost, and it is not obvious that topologically invariant information about the knot can be extracted.

6.1. A Unitary Representation of the Three Strand Braid Group and the Corresponding Quantum Computer. Many questions are raised by the formulation of a quantum computer associated with a given Morse link 
diagram. First of all, unitary solutions to the Yang-Baxter equation (or unitary representations of the Artin braid group) that also give link invariants are not so easy to come by. We gave a small example of a unitary representation of the threestrand braid group in the first section of this paper. Thus we, are prepared to look at some aspects of the computation of a knot invariant as a quantum computation. In fact, we can use this representation to compute the Jones polynomial for closures of 3-braids, and therefore this representation provides a test case for the corresponding quantum computation. We now analyze this case by first making explicit how the bracket polynomial is computed from this representation.

First recall that the representation depends on two matrices $U_{1}$ and $U_{2}$ with

$$
U_{1}=\left[\begin{array}{ll}
\delta & 0 \\
0 & 0
\end{array}\right]
$$

and

$$
U_{2}=\left[\begin{array}{cc}
\delta^{-1} & \sqrt{1-\delta^{-2}} \\
\sqrt{1-\delta^{-2}} & \delta-\delta^{-1}
\end{array}\right]
$$

The representation is given on the two braid generators by

$$
\begin{aligned}
& \rho\left(\sigma_{1}\right)=A I+A^{-1} U_{1} \\
& \rho\left(\sigma_{2}\right)=A I+A^{-1} U_{2}
\end{aligned}
$$

for any $A$ with $\delta=-A^{2}-A^{-2}$, and with $A=e^{i \theta}$, then $\delta=-2 \cos (2 \theta)$. We get the specific range of angles $|\theta| \leq \pi / 6$ and $|\theta-\pi| \leq \pi / 6$ that give unitary representations of the three-strand braid group.

Note that $\operatorname{tr}\left(U_{1}\right)=\operatorname{tr}\left(U_{2}\right)=\delta$ while $\operatorname{tr}\left(U_{1} U_{2}\right)=\operatorname{tr}\left(U_{2} U_{1}\right)=1$. If $b$ is any braid, let $I(b)$ denote the sum of the exponents in the braid word that expresses $b$. For $b$ a three-strand braid, it follows that

$$
\rho(b)=A^{I(b)} I+\tau(b)
$$

where $I$ is the $2 \times 2$ identity matrix and $\tau(b)$ is a sum of products in the Temperley Lieb algebra involving $U_{1}$ and $U_{2}$. Since the Temperley Lieb algebra in this dimension is generated by $I, U_{1}, U_{2}, U_{1} U_{2}$ and $U_{2} U_{1}$, it follows that

$$
<\bar{b}>=A^{I(b)} \delta^{2}+\operatorname{tr}(\tau(b))
$$

where $\bar{b}$ denotes the standard braid closure of $b$, and the sharp brackets denote the bracket polynomial as described in previous sections. From this we see at once that

$$
<\bar{b}>=\operatorname{tr}(\rho(b))+A^{I(b)}\left(\delta^{2}-2\right) .
$$

It follows from this calculation that the question of computing the bracket polynomial for the closure of the three-strand braid $b$ is mathematically equivalent to the problem of computing the trace of the matrix $\rho(b)$. To what extent can our quantum computer determine the trace of this matrix?

The matrix in question is a product of unitary matrices, the quantum gates that we have associated with the braids $\sigma_{1}$ and $\sigma_{2}$. The entries of the matrix $\rho(b)$ 
are the results of preparation and detection for the two dimensional basis of qubits for our machine:

$$
<i|\rho(b)| j>.
$$

Given that the computer is prepared in $|j\rangle$, the probability of observing it in state $|i\rangle$ is equal to $|\langle i|\rho(b)| j\rangle|^{2}$. Thus we can, by running the quantum computation repeatedly, estimate the absolute squares of the entries of the matrix $\rho(b)$. This will not yield the complex phase information that is needed for either the trace of the matrix or the absolute value of that trace. Thus we conclude that our quantum computer can compute information relating to the braiding process, but that it cannot approximate the full value of the bracket polynomial.

Note that our quantum computer does indeed have the capability to detect three strand braiding, since for a braid $b$ the matrix $\rho(b)$ can have non-trivial offdiagaonal elements. The absolute squares of these elements are approximated by successive runs of the quantum computer. In this quantum computer, braiding corresponds to entangled quantum states and is delectable by that token. The bracket polynomial itself depends upon subtler phase relationships and is not detectable by this quantum computer.

6.2. Comments. These results are less than satisfying since there does not seem to be a way to calculate the entire knot polynomial even by probabilisitic approximations. It is not clear what the practical value of such a computation will be for understanding a given link invariant. Nevertheless, it is to be expected that a close relationship between quantum link invariants and quantum computing will be fruitful for both fields.

There are other ideas in the topology that deserve comparison with the quantum states. For example, topological entanglement in the sense of linking and braiding is intuitively related to the entanglement of quantum states. In our general model using a unitary representation of the braid group, topological entanglement entails quantum entanglement. The quantum topological states associated with the bracket polynomial would certainly figure strongly in a quantum computing model of this algorithm. The specific model that we have given uses only one qubit and so does not produce entanglement. There are other representations of the Artin Braid group that do produce quantum entanglements corresponding to topological braiding. These phenomena will be the subject of a subsequent paper [20].

We mention one further possibility. In the paper [21] by Lidar and Biham the authors show how to simulate special cases of the Ising model on a quantum computer. Their method is more combinatorial and less algebraic than the approach sketched in this section using braiding. It is possible that a generalization of their approach will work for the state sum of the bracket polynomial. This is a topic for further research.

6.3. And Quantum Field Theory. Finally, it is important to remark that there is an interpretation of the Jones polynomial in terms of quantum field theory. Witten [26] writes down a functional integral for link invariants in a 3-manifold $M$ : 


$$
Z(M, K)=\int d A \exp [(i k / 4 \pi) S(M, A)] \operatorname{tr}\left(\operatorname{Pexp}\left(\int_{K} A\right)\right) .
$$

Here $M$ denotes a 3 -manifold without boundary and $A$ is a gauge field (gauge connection) defined on $M$. The gauge field is a one-form on $M$ with values in a representation of a Lie algebra. $S(M, A)$ is the integral over $M$ of the trace of the Chern-Simons three-form $C S=A d A+(2 / 3) A A A$. (The product is the wedge product of differential forms.)

With the standard representation of the Lie algebra of $S U(2)$ as $2 \times 2$ complex matrices, one can see that the formalism of $Z(S, K)\left(S^{3}\right.$ denotes the threedimensional sphere.) yields the Jones polynomial with the basic properties as we have discussed. See Witten's paper or $[\mathbf{2 6}]$ or $[\mathbf{1 5}],[\mathbf{1 6}]$.

The question is: How does the quantum field theory approach to the Jones polynomial relate to quantum computing? One way to discuss this question is to reformulate (topological) quantum field theories as state summations, as we did for the Jones polynomial, and then proceed in a fashion analogous to our amplitudes discussion above. It is more challenging to try to imagine reformulating quantum computing at the level of quantum field theory. If this were accomplished, the subject of quantum computing and the Jones polynomial might well take a new road.

\section{Summary}

In relating quantum computing with knot polynomials the key themes are unitarity and measurement. Much is now surely unforeseen. For a good survey of quantum computing we recommend $[\mathbf{1}]$ and $[\mathbf{2 2}]$, and for another view of topological issues see $[\mathbf{6}]$ and $[\mathbf{7}]$. See $[\mathbf{2 5}]$ for an excellent treatment of measurement theory in quantum mechanics and a usage of the Dirac formalism that is in resonance with the concerns of this paper.

\section{References}

[1] D. Aharonov. Quantum computation. quant-phys/9812037 15Dec 1998.

[2] M.F. Atiyah. The Geometry and Physics of Knots. Cambridge University Press (1990).

[3] R.J. Baxter. Exactly Solved Models in Statistical Mechanics. Acad. Press (1982).

[4] P.A.M. Dirac. Principles of Quantum Mechanics. Oxford University Press (1958).

[5] R. Feynman and A.R. Hibbs. Quantum Mechanics and Path Integrals. McGraw Hill (1965).

[6] M. Freedman. Topological Views on Computational Complexity. Documenta Mathematica Extra Volume ICM (1998), pp. 453-464.

[7] M. Freedman, M. Larsen and Z. Wang. A modular functor which is universal for quantum computation. arXiv:quant-ph/0001108v2 1 Feb 2000.

[8] V.F.R.Jones.A polynomial invariant for links via von Neumann algebras. Bull.Amer.Math.Soc. 129 (1985) 103-112.

[9] D.S.Freed and R.E.Gompf. Computer Calculation of Witten's 3-Manifold Invariant. Commun. Math. Phys. 141 (1991), pp. 79-117.

[10] V.F.R.Jones. A new knot polynomial and von Neumann algebras. Notices of AMS 33 (1986) 219-225.

[11] L.H.Kauffman. State Models and the Jones Polynomial. Topology 26 (1987) 395-407.

[12] L.H. Kauffman. On Knots. Annals of Mathematics Studies Number 115, Princeton University Press (1987). 
[13] L.H.Kauffman. New invariants in the theory of knots. Amer. Math. Monthly Vol.95,No.3,March 1988. pp 195-242.

[14] L.H.Kauffman. Statistical mechanics and the Jones polynomial. AMS Contemp. Math. Series (1989), Vol. 78. pp. 263-297.

[15] L.H. Kauffman. Knots and Physics, World Scientific Pub. (1991 and 1993).

[16] L. H. Kauffman. Functional integration and the theory of knots. J. Math. Phys. Vol. 36, No.5 (19950, pp. 2402-2429.

[17] L. H. Kauffman. Witten's Integral and the Kontsevich Integral. In Particles Fields and Gravitation. edited by Jakub Rembielinski, AIP Proceedings No. 453, American Inst. of Physics Pub. (1998), pp. 368 - 381.

[18] L.H.Kauffman (Editor). Knots and Applications, World Scientific Pub. Co. (1995).

[19] L.H.Kauffman. Knots and Statistical Mechanics, Proceedings of Symposia in Applied Mathematics - The Interface of Knots and Physics (edited by L. Kauffman), Vol.51 (1996), pp. $1-87$.

[20] L.H.Kauffman and S. Lomonaco. Topological Entanglement and Quantum Entanglement. (in preparation)

[21] D. Lidar and O. Biham. Simulating ising spin glasses on a quantum computer. quantph/9611038v6 23 Sept. 1997.

[22] S. Lomonaco. A rosetta stone for quantum mechanics with an introduction to quantum computiation. quant-ph/0007045 (2000).

[23] K. Reidemeister. Knotentheorie. Chelsea, New York (1948), Julius Springer (1932).

[24] N.Yu.Reshetikhin and V.G. Turaev. Invariants of 3-manifolds via link polynomials and quantum groups. Invent. Math. Vol. 103 (1991), pp. 547-597.

[25] J. Schwinger. Quantum Mechanics: Symbolism of Atomic Measurement. Springer-Verlag, 2001.

[26] E. Witten. Quantum field theory and the Jones polynomial. Commun.Math.Phys. 121, 351399 (1989).

(Louis H. Kauffman) Department of Mathematics, Statistics, and Computer Science,

University of Illinois at Chicago, 851 South Morgan Street, Chicago, IL 60607-7045

E-mail address, Louis H. Kauffman: kauffman@uic.edu

$U R L:$ http://math.uic.edu/ ₹kauffman 